\title{
In vivo evaluation of the effects of simultaneous inhibition of GLUT-1 and HIF-1a by antisense oligodeoxynucleotides on the radiosensitivity of laryngeal carcinoma using micro ${ }^{18}$ F-FDG PET/CT
}

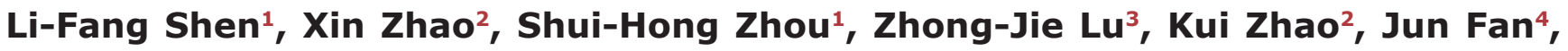 \\ Min-Li Zhou ${ }^{1}$ \\ ${ }^{1}$ Department of Otolaryngology, The First Affiliated Hospital, College of Medicine, Zhejiang University, Hangzhou City, \\ Zhejiang Province, China \\ ${ }^{2}$ Center of PET/CT, The First Affiliated Hospital, College of Medicine, Zhejiang University, Hangzhou City, Zhejiang Province, \\ China \\ ${ }^{3}$ Department of Radiotherapy, The First Affiliated Hospital, College of Medicine, Zhejiang University, Hangzhou City, Zhejiang \\ Province, China \\ ${ }^{4}$ State Key Laboratory for Diagnosis and Treatment of Infectious Diseases, The First Affiliated Hospital, College of Medicine, \\ Zhejiang University, Hangzhou City, Zhejiang Province, China \\ Correspondence to: Shui-Hong Zhou, email: zhouyunzhoush@163.com \\ Keywords: hypoxia-inducible factor 1a, glucose transporter-1, antisense oligodeoxynucleotides, radiosensitivity, ${ }^{18}$ F-FDG \\ micro PET/CT \\ Received: November 04, $2016 \quad$ Accepted: March 15, 2017 Published: March 29, 2017 \\ Copyright: Shen et al. This is an open-access article distributed under the terms of the Creative Commons Attribution License (CC-BY), \\ which permits unrestricted use, distribution, and reproduction in any medium, provided the original author and source are credited.
}

\section{ABSTRACT}

Purpose: Hypoxia-inducible factor 1a (HIF-1a) and glucose transporter-1 (GLUT-1) are two important hypoxic markers associated with the radioresistance of cancers including laryngeal carcinoma. We evaluated whether the simultaneous inhibition of GLUT-1 and HIF-1a expression improved the radiosensitivity of laryngeal carcinoma. We explored whether the expression of HIF-1a and GLUT-1 was correlated with 2'-deoxy-2'-[18F]fluoro-D-glucose (18F-FDG) uptake and whether 18F-FDG positron emission tomography-computed tomography (PET/CT) was appropriate for early evaluation of the response of laryngeal carcinoma to targeted treatment in vivo.

Materials and Methods: To verify the above hypotheses, an in vivo model was applied by subcutaneously injecting Hep-2 $(2 \times 107 / \mathrm{mL} \times 0.2 \mathrm{~mL})$ and Tu212 cells $(2 \times 107 / \mathrm{mL} \times 0.2 \mathrm{~mL})$ into nude mice. The effects of HIF-1a antisense oligodeoxynucleotides (AS-ODNs) $(100 \mu \mathrm{g})$ and GLUT-1 AS-ODNs (100 $\mu \mathrm{g})$ on the radiosensitivity of laryngeal carcinoma were assessed by tumor volume and weight, microvessel density (MVD), apoptosis index (AI) and necrosis in vivo based on a full factorial $\left(2^{3}\right)$ design. ${ }^{18}$ F-FDG-PET/CT was taken before and after the treatment of xenografts. The relationships between HIF-1a and GLUT-1 expression and ${ }^{18}$ F-FDG uptake in xenografts were estimated and the value of ${ }^{18} \mathrm{~F}-\mathrm{FDG}-\mathrm{PET} / \mathrm{CT}$ was assessed after treating the xenografts.

Results: 10 Gy X-ray irradiation decreased the weight of Hep-2 xenografts 8 and 12 days after treatment, and the weights of Tu212 xenografts 8 days after treatment. GLUT-1 AS-ODNs decreased the weight of Tu212 xenografts 12 days after treatment. There was a synergistic interaction among the three treatments (GLUT-1 AS-ODNs, HIF-1a AS-ODNs and 10Gy $X$-ray irradiation) in increasing apoptosis, decreasing MVD, and increasing necrosis in Hep-2 xenografts 8 days after treatment $(p<0.05)$ and in Tu212 xenografts 12 days after treatment $(p<0.001)$. Standardized uptake value (tumor/normal tissue)( SUVmaxT/N) did not show a statistically significant correlation with GLUT1 and HIF-1a expression and therapeutic effect (necrosis, apoptosis). 


\section{Conclusions: Simultaneous inhibition of HIF-1a and GLUT-1 expression might increase the radiosensitivity of laryngeal carcinoma, decreasing MVD, and promoting apoptosis and necrosis. ${ }^{18} \mathrm{~F}-\mathrm{FDG}-\mathrm{PET} / \mathrm{CT}$ wasn't useful in evaluating the therapeutic effect on laryngeal cancer in this animal study.}

\section{INTRODUCTION}

Although promising therapeutic strategies have been described, the poor overall survival rate of patients with laryngeal carcinoma remains unchanged [1]. One possible cause may be radioresistance of laryngeal carcinomas. The underlying mechanisms of radioresistance is still unclear and involves multiple factors including tumor cell proliferation, hypoxia and intrinsic radioresistance $[2,3]$.

Among them, hypoxia is an important issue [4]. HIF- $1 \alpha$ is important factor induced during the adaptive response to hypoxia [5]. HIF-1 $\alpha$ regulates multiple aspects of tumorigenesis, including proliferation, differentiation, angiogenesis, metabolism, metastasis, and responses to radiation therapy, making it a key regulator of malignant tumor phenotypes [6,7]. HIF-1 $\alpha$ has been associated with a poor prognosis in laryngeal carcinoma [8]. It has been reported that high expression of HIF and other endogenous hypoxia-related proteins was associated with radioresistance and a worse overall survival rate $[5,9]$. Accordingly, HIF-1 $\alpha$ has been suggested as a potential therapeutic target to improve radiosensitivity in vitro and in vivo $[10,11]$.

To our knowledge, there is only one report on targeting HIF-1 $\alpha$ to enhance radiosensitivity in laryngeal cancer [12]. However, it was an in vitro study and the HIF-1 $\alpha$ inhibitor used was not specific. Thus, the role of HIF-1 $\alpha$ in laryngeal carcinoma radioresistance and whether inhibition of HIF- $1 \alpha$ expression can improve radiosensitivity of laryngeal carcinoma require further evaluation.

At least one study has shown the limitations associated with inhibiting HIF-1 $\alpha$ alone to improve radiosensitivity [13], thus, more effective strategies to enhance the radiosensitivity of laryngeal carcinoma need to be investigated. It may be useful to inhibit HIF-1 downstream target genes, including GLUT-1. GLUT-1 has been considered a possible intrinsic marker of hypoxia in malignant tumors, including laryngeal carcinoma $[14,15]$ Some studies have also demonstrated that increased GLUT-1 expression was associated with radioresistance $[16,17]$. Our previous findings showed that GLUT-1 AS-ODNs inhibited glucose uptake and the proliferation of Hep-2 cells [18], and that GLUT-1 over-expression was associated with radioresistance in laryngeal cancer, furthermore, suppressing the expression of GLUT-1 may enhance the radioresistance of laryngeal carcinoma [19]. These results suggest that GLUT-1 expression is a marker of radioresistance in malignant tumors.

Although Amann et al proposed that combined inhibition of HIF-1 $\alpha$ and GLUT-1 may be a novel therapeutic stategy in hepatocellular carcinoma [20], there is no report on the simultaneous inhibition of HIF-1 $\alpha$ and GLUT-1 in laryngeal cancer. In this study, we assessed the effect of simultaneous inhibition of HIF- $1 \alpha$ and GLUT-1 expression on radioresistance in laryngeal carcinomas in vivo. $2^{3}$ factorial design is adopted in this study concerning with the effects of formulation variables and their interactions on response variables to obtain the optimized formulation.

PET is a quantitative molecular imaging technique that allows noninvasive imaging in vivo and quantification of biological processes [21]. A microPET/CT scanner for animal studies has provided a novel technology for molecular imaging assays of metabolism and signal transduction [22].

The relationships between GLUT1, HIF1 $\alpha$ expression and ${ }^{18} \mathrm{~F}$-FDG uptake in head and neck squamous cell carcinoma(HNSCC) remain controversial. In our previous study, the expression of GLUT1 and HIF $1 \alpha$ was significantly correlated with ${ }^{18} \mathrm{~F}$-FDG uptake in patients with laryngeal carcinoma [23]. However, Mason et al reported that ${ }^{18} \mathrm{~F}$-FDG uptake in HNSCC xenografts might not reflect the level of metabolic activity characteristic of HNSCC [24].

A few studies have investigated whether micro PET/CT is useful for determining radiosensitivity in nasopharyngeal carcinoma [25], and human glioblastoma [26] in vivo. In this study, we evaluated the radiosensitivity of laryngeal carcinoma using micro PET/CT and assessed the relationships between HIF1 $\alpha$, GLUT1-1 expression and 18F-FDG uptake in vivo.

\section{RESULTS}

\section{General observations, volume, weight of the Hep-2 and Tu212 xenografts}

The volumes of Hep-2 and Tu212 xenografts reached $100 \mathrm{~mm}^{3}$ at 9 days and 11 days after inoculation respectively. Mice in each group exhibited no obvious abnormalities in mental behavior, eating habits, defecation, weight or mortality during the experimental period.

Mice were sacrificed 8 days after treatment initiation. The main effect of 10 Gy X-ray irradiation on tumor weight was $0.158 \pm 0.03 \mathrm{~g}(p=0.002)$ in Hep-2 xenografts, $0.05 \pm 0.016 \mathrm{~g}(p=0.038)$ in Tu212 xenografts, respectively (Table 1 ). At 12 days after treatment, the main effect of $10 \mathrm{~Gy} \mathrm{X}$-ray- irradiation on tumor weight was $0.208 \pm 0.058 \mathrm{~g}$ in Hep-2 xenografts $(p=0.022)$, and the main effect of GLUT-1 AS-ODNs was 
Table 1: Observed responses of xenografts 8 days after treatment in the $2^{3}$ factorial design with three independent parameters and their two levels (GLUT-1 AS-ODNs and HIF-1 AS-ODNs $100 \mu \mathrm{g}$ or $0 \mu \mathrm{g}$, X-ray irradiation $10 \mathrm{~Gy}$ or $0 \mathrm{~Gy})$

\begin{tabular}{|c|c|c|c|c|c|c|c|c|c|c|c|}
\hline \multirow{2}{*}{$\mathbf{G}$} & \multirow{2}{*}{$A(\mu g)$} & \multirow{2}{*}{ B $(\mu \mathrm{g})$} & \multirow{2}{*}{ C (Gy) } & \multicolumn{2}{|c|}{ Tumor weight $^{x}(\mathrm{~g})$} & \multicolumn{2}{|c|}{ AI $(\%)^{x}$} & \multicolumn{2}{|c|}{ MVD $^{x}$} & \multicolumn{2}{|c|}{ Necrosis ratex $(\%)$} \\
\hline & & & & Нер-2 & Tu212 & Нер-2 & Tu212 & Нер-2 & Tu212 & Нер-2 & Tu212 \\
\hline G1 & 100 & 100 & 10 & $0.36 \pm 0.03$ & $0.11 \pm 0.01$ & $47.71 \pm 3.38$ & $38.10 \pm 0.41$ & $3.67 \pm 2.10$ & $1.33 \pm 0.66$ & $51.67 \pm 7.26$ & $63.33 \pm 3.33$ \\
\hline G2 & 100 & 100 & 0 & $0.27 \pm 0.08$ & $0.13 \pm 0.02$ & $30.45 \pm 2.51$ & $31.66 \pm 0.71$ & $6.00 \pm 1.10$ & $6.33 \pm 0.88$ & $10.00 \pm 2.88$ & $23.33 \pm 6.01$ \\
\hline G3 & 0 & 100 & 10 & $0.57 \pm 0.06$ & $0.10 \pm 0.01$ & $8.67 \pm 1.35$ & $9.70 \pm 1.03$ & $4.67 \pm 0.88$ & $4.33 \pm 0.33$ & $8.33 \pm 1.66$ & $23.33 \pm 1.66$ \\
\hline G4 & 100 & 0 & 10 & $0.41 \pm 0.09$ & $0.09 \pm 0.01$ & $14.04 \pm 2.19$ & $17.72 \pm 1.41$ & $11.33 \pm 1.85$ & $10.67 \pm 1.33$ & $10.00 \pm 5.00$ & $40.00 \pm 2.88$ \\
\hline G5 & 0 & 0 & 10 & $0.25 \pm 0.03$ & $0.11 \pm 0.02$ & $4.26 \pm 0.53$ & $5.68 \pm 1.61$ & $15.67 \pm 1.76$ & $12.00 \pm 3.00$ & $8.33 \pm 1.66$ & $35.00 \pm 7.63$ \\
\hline G6 & 0 & 100 & 0 & $0.21 \pm 0.06$ & $0.18 \pm 0.06$ & $6.07 \pm 0.04$ & $7.75 \pm 1.05$ & $13.00 \pm 1.15$ & $11.67 \pm 1.76$ & $5.00 \pm 0.00$ & $15.00 \pm 5.00$ \\
\hline G7 & 100 & 0 & 0 & $0.16 \pm 0.01$ & $0.13 \pm 0.02$ & $10.41 \pm 0.51$ & $14.85 \pm 1.27$ & $16.33 \pm 2.18$ & $18.33 \pm 2.72$ & $10.00 \pm 2.88$ & $5.00 \pm 0.00$ \\
\hline G8 & 0 & 0 & 0 & $0.33 \pm 0.04$ & $0.16 \pm 0.04$ & $2.24 \pm 0.11$ & $4.74 \pm 1.28$ & $34.00 \pm 2.64$ & $34.00 \pm 3.05$ & $1.66 \pm 1.66$ & $0.00 \pm 0.00$ \\
\hline \multicolumn{4}{|c|}{ a ( $p$-value) } & 0.427 & 0.275 & $<0.001$ & $<0.001$ & $<0.001$ & $<0.001$ & $<0.001$ & $<0.001$ \\
\hline \multicolumn{4}{|c|}{$\mathrm{b}$ ( $p$-value) } & 0.09 & 0.711 & $<0.001$ & $<0.001$ & $<0.001$ & $<0.001$ & $<0.001$ & 0.591 \\
\hline \multicolumn{4}{|c|}{$\mathrm{c}(p$-value $)$} & 0.002 & 0.038 & $<0.001$ & 0.003 & $<0.001$ & $<0.001$ & $<0.001$ & $<0.001$ \\
\hline \multicolumn{4}{|c|}{$\mathrm{ab}(p$-value $)$} & 0.346 & 0.941 & $<0.001$ & $<0.001$ & 0.015 & 0.145 & 0.002 & 0.318 \\
\hline \multicolumn{4}{|c|}{ ac ( $p$-value) } & 0.83 & 0.379 & 0.005 & 0.047 & 0.002 & 0.009 & 0.007 & $<0.001$ \\
\hline \multicolumn{4}{|c|}{ bc ( $p$-value $)$} & 0.156 & 1.000 & 0.012 & 0.245 & 0.026 & 0.007 & 0.002 & 0.003 \\
\hline \multicolumn{4}{|c|}{ abc ( $p$-value) } & 0.13 & 0.555 & 0.018 & 0.344 & 0.174 & 0.05 & $<0.001$ & 0.004 \\
\hline
\end{tabular}

G: group, A: GLUT-1 AS-ODNs, B: HIF-1 $\alpha$ AS-ODNs,C: X-ray irradiation.

a: main effect of GLUT-1 AS-ONDs, b: main effect of HIF-1 $\alpha$ AS-ODNs, c: main effect of X-ray irradiation, ab: interaction effect between GLUT-1 AS-ONDs and HIF-1 $\alpha$ ASODNs, ac: interaction effect between GLUT-1 AS-ONDs and X-ray irradiation, bc: interaction effect between HIF-1 $\alpha$ AS-ODNs and X-ray irradiation, abc: interaction effect between GLUT-1 AS-ONDs and HIF-1 $\alpha$ AS-ODNs and X-ray irradiation.

${ }^{\mathrm{x}}$ Mean $\pm \operatorname{Std}($ standard deviation).

$0.115 \pm 0.025 \mathrm{~g}$ in Tu212 xenografts $(p=0.006)$ (Table 2$)$. GLUT-1 AS-ODNs, HIF-1 $\alpha$ AS-ODNs and 10Gy X-ray irradiation showed no interaction effects or main effects on the volumes of Hep-2 or Tu212 xenografts $(P>0.05)$ (Figure 1).

\section{Effects of GLUT-1 AS-ODNs, HIF-1a AS-ODNs and 10Gy $\mathrm{X}$-ray irradiation on xenografts GLUT-1 and HIF-1 $\alpha$ mRNA levels}

There was a synergistic interaction effect of the three treatments combined GLUT-1 AS-ODNs, HIF-1 $\alpha$ AS-ODNs and 10Gy X-ray irradiation on decreasing the expression of GLUT-1 mRNA in Hep-2 and Tu212 xenografts 8 days and 12 days after treatment $(p<0.01)$. Only HIF-1 $\alpha$ AS-ODNs decreased HIF-1 $\alpha$ mRNA expression in Hep-2 xenografts significantly 8 days after treatment $(p<0.001)$. There were synergistic interaction effects of GLUT-1 AS-ODNs combined with HIF-1 $\alpha$ AS-ODNs, GLUT-1 AS-ODNs combined with 10Gy X-ray irradiation, HIF-1 $\alpha$ AS-ODNs combined with 10 Gy X-ray irradiation on decreasing the expression of HIF-1 $\alpha$ mRNA in Hep-2 xenografts significantly 12 days after treatment $(P=0.021, p=0.001$, $p<0.001$, respectively). In Tu212 xenografts, HIF-1 $\alpha$ AS-ODNs and 10 Gy X-ray irradiation had synergistic interaction effects on decreasing the expression of HIF-1 $\alpha$
mRNA 8 days and 12 days after treatment $(p=0.026,0.004$, respectively) (Table 3) (Table 4).

\section{Effects of GLUT-1 AS-ODNs, HIF-1 $\alpha$ AS-ODNs and 10Gy $X$-ray irradiation on xenografts GLUT-1 and HIF-1 $\alpha$ protein levels}

There was a synergistic interaction effect of the three treatments combined GLUT-1 AS-ODNs, HIF-1 $\alpha$ AS-ODNs and 10Gy X-ray irradiation on decreasing the expression of GLUT-1 protein in Hep-2 and Tu212 xenografts 8 days after treatment ( $p=0.002, p=0.041$, respectively). HIF-1 $\alpha$ AS-ODNs and 10 Gy X-ray irradiation both decreased GLUT-1 protein expression in Hep- 2 cells 12 days after treatment $(p=0.036,0.017$, respectively), surprisingly, GLUT-1 AS-ODNs alone didn't reduce the GLUT-1 protein levels. In Tu212 xenografts, GLUT-1 AS-ODNs, HIF-1 $\alpha$ AS-ODNs or 10 Gy X-ray irradiation alone decreased GLUT-1 protein expression significantly ( $p=0.001,0.004,0.006$, respectively) 12 days after treatment, but there was no interaction effect. There was a synergistic interaction effect among the three treatments on decreasing HIF-1 $\alpha$ protein levels in Hep-2 xenografts 8 days after treatment $(p=0.002)$. There was no equivalent synergistic interaction effect in Tu212 xenografts 8 days after treatment, or in Hep-2 or 
Table 2: Observed responses of xenografts 12 days after treatment in the $2^{3}$ factorial design with three independent parameters and their two levels (GLUT-1 AS-ODNs and HIF-1 $\alpha$ AS-ODNs $100 \mu \mathrm{g}$ or $0 \mu \mathrm{g}$, X-ray irradiation $10 \mathrm{~Gy}$ or $0 \mathrm{~Gy}$ )

\begin{tabular}{|c|c|c|c|c|c|c|c|c|c|c|c|}
\hline \multirow{2}{*}{ G } & \multirow{2}{*}{$A(\mu g)$} & \multirow{2}{*}{ B $(\mu \mathrm{g})$} & \multirow{2}{*}{ C (Gy) } & \multicolumn{2}{|c|}{ Tumor weight $^{\mathrm{x}}(\mathrm{g})$} & \multicolumn{2}{|c|}{ AI $(\%)^{x}$} & \multicolumn{2}{|c|}{ MVD $^{x}$} & \multicolumn{2}{|c|}{ Necrosis rate $(\%)^{x}$} \\
\hline & & & & Нер-2 & Tu212 & Hep-2 & Tu212 & Нер-2 & Tu212 & Нер-2 & Tu212 \\
\hline G1 & 100 & 100 & 10 & $0.14 \pm 0.02$ & $0.25 \pm 0.04$ & $14.33 \pm 0.67$ & $27.24 \pm 0.36$ & $2.33 \pm 1.33$ & $4.00 \pm 0.57$ & $56.67 \pm 3.33$ & $46.67 \pm 6.66$ \\
\hline $\mathrm{G} 2$ & 100 & 100 & 0 & $0.47 \pm 0.02$ & $0.21 \pm 0.05$ & $18.99 \pm 1.13$ & $17.19 \pm 1.16$ & $9.33 \pm 0.33$ & $10.00 \pm 0.57$ & $11.67 \pm 3.33$ & $36.67 \pm 8.81$ \\
\hline G3 & 0 & 100 & 10 & $0.42 \pm 0.12$ & $0.30 \pm 0.06$ & $4.32 \pm 0.03$ & $6.42 \pm 0.18$ & $7.00 \pm 1.00$ & $7.66 \pm 0.33$ & $5.00 \pm 0.00$ & $40.00 \pm 15.28$ \\
\hline G4 & 100 & 0 & 10 & $0.26 \pm 0.04$ & $0.26 \pm 0.05$ & $4.82 \pm 0.06$ & $11.09 \pm 0.30$ & $16.67 \pm 2.66$ & $13.33 \pm 0.88$ & $10.00 \pm 0.00$ & $43.33 \pm 8.18$ \\
\hline G5 & 0 & 0 & 10 & $0.24 \pm 0.04$ & $0.33 \pm 0.06$ & $2.84 \pm 0.15$ & $3.88 \pm 0.57$ & $10.33 \pm 1.20$ & $14.00 \pm 0.57$ & $5.00 \pm 0.00$ & $23.33 \pm 8.33$ \\
\hline G6 & 0 & 100 & 0 & $0.53 \pm 0.22$ & $0.30 \pm 0.01$ & $4.03 \pm 0.18$ & $5.78 \pm 0.98$ & $15.33 \pm 0.88$ & $16.00 \pm 0.57$ & $11.67 \pm 3.33$ & $16.67 \pm 3.33$ \\
\hline G7 & 100 & 0 & 0 & $0.60 \pm 0.20$ & $0.18 \pm 0.04$ & $8.24 \pm 0.35$ & $9.03 \pm 0.49$ & $25.33 \pm 2.40$ & $27.67 \pm 1.45$ & $5.00 \pm 0.00$ & $11.67 \pm 1.66$ \\
\hline G8 & 0 & 0 & 0 & $0.59 \pm 0.02$ & $0.42 \pm 0.06$ & $1.78 \pm 0.21$ & $2.73 \pm 0.71$ & $41.33 \pm 1.45$ & $45.00 \pm 2.88$ & $5.00 \pm 0.00$ & $5.00 \pm 0.00$ \\
\hline \multicolumn{4}{|c|}{ a ( $p$-value) } & 0.952 & 0.006 & $<0.001$ & $<0.001$ & $<0.001$ & $<0.001$ & $<0.001$ & 1.000 \\
\hline \multicolumn{4}{|c|}{$\mathrm{b}$ ( $p$-value $)$} & 0.592 & 0.379 & $<0.001$ & $<0.001$ & $<0.001$ & $<0.001$ & $<0.001$ & 0.043 \\
\hline \multicolumn{4}{|c|}{$\mathrm{c}(p$-value $)$} & 0.022 & 0.837 & $<0.001$ & $<0.001$ & $<0.001$ & $<0.001$ & $<0.001$ & 1.000 \\
\hline \multicolumn{4}{|c|}{$\mathrm{ab}$ ( $p$-value) } & 0.051 & 0.292 & $<0.001$ & $<0.001$ & $<0.001$ & 0.032 & $<0.001$ & 0.018 \\
\hline \multicolumn{4}{|c|}{ ac ( $p$-value) } & 0.134 & 0.140 & $<0.001$ & $<0.001$ & $<0.001$ & $<0.001$ & $<0.001$ & 0.205 \\
\hline \multicolumn{4}{|c|}{ bc ( $p$-value) } & 0.873 & 0.767 & 0.173 & $<0.001$ & $<0.001$ & $<0.001$ & $<0.001$ & 0.162 \\
\hline \multicolumn{4}{|c|}{ abc ( $p$-value) } & 0.826 & 0.356 & 0.747 & $<0.001$ & $<0.001$ & $<0.001$ & $<0.001$ & 0.041 \\
\hline
\end{tabular}

G: group, A: GLUT-1 AS-ODNs, B: HIF-1 $\alpha$ AS-ODNs,C: X-ray irradiation.

a: main effect of GLUT-1 AS-ONDs, b: main effect of HIF-1 $\alpha$ AS-ODNs, c: main effect of X-ray irradiation, ab: interaction effect between GLUT-1 AS-ONDs and HIF-1 $\alpha$ ASODNs, ac: interaction effect between GLUT-1 AS-ONDs and X-ray irradiation, bc: interaction effect between HIF-1 $\alpha$ AS-ODNs and X-ray irradiation, abc: interaction effect between GLUT-1 AS-ONDs and HIF-1 $\alpha$ AS-ODNs and X-ray irradiation.

${ }^{\mathrm{x}}$ Mean $\pm \operatorname{Std}($ standard deviation).

Tu212 xenografts 12 days after treatment. Only HIF-1 $\alpha$ AS-ODNs alone significantly decreased HIF- $1 \alpha$ protein levels in Tu212 cells 8 days after treatment $(p=0.003)$, HIF-1 $\alpha$ AS-ODNs alone and 10Gy X-ray irradiation alone significantly decreased HIF- $1 \alpha$ protein levels in Hep-2 xenografts ( $p<0.001,0.014$, respectively) and Tu212 xenografts ( $p=0.001,0.006$, respectively) 12 days after treatment. (Figure 2) (Table 3) (Table 4).

\section{Effects of GLUT-1 AS-ODNs, HIF-1 $\alpha$ AS-ODNs and 10Gy X-ray irradiation on apoptosis, MVD, and necrosis in the xenografts}

TUNEL positive staining showed that the nucleus was brown or brown-yellow, that is, apoptotic cells (Figure 3) (Figure 4). There was a synergistic interaction effect of the three treatments combined GLUT-1 ASODNs and HIF-1 $\alpha$ AS-ODNs and 10Gy X-ray irradiation on increasing apoptosis in Hep-2 cells 8 days after treatment $(p=0.018)$. Although there was no synergistic interaction effect of the three treatments on apoptosis in Tu212 xenografts, GLUT-1 AS-ODNs combined with HIF-1 $\alpha$ AS-ODNs, GLUT-1 AS-ODNs combined with 10Gy X-ray irradiation had synergistic interaction effects on increasing apoptosis in Tu212 xenografts 8 days after treatment $(P<0.001, p=0.047$, respectively) (Table 1). Interestingly, there was a synergistic interaction effect of the three treatments combined on increasing apoptosis in Tu212 cells 12 days after treatment $(p<0.001)$, there was no such effect on Hep-2 cells 12 days after treatment $(p=0.747)$. GLUT-1 AS-ODNs combined with HIF-1 $\alpha$ AS-ODNs, GLUT-1 AS-ODNs combined with 10Gy $\mathrm{X}$-ray irradiation had synergistic interaction effects on increasing apoptosis in Hep-2 xenografts 12 days after treatment $(P<0.001, P<0.001$, respectively) (Table 2$)$.

CD34 is the most sensitive marker of vascular endothelial cells, positive staining showed cytoplasm was light brown to brown (Figure 5) (Figure 6). There was a synergistic interaction effect of the three treatments combined GLUT-1 AS-ODNs and HIF- $1 \alpha$ AS-ODNs and 10 Gy X-ray irradiation on decreasing MVD in Tu212 xenografts 8 days after treatment $(p=0.05)$, and in Hep-2 and Tu212 xenografts 12 days after treatment $(P<0.001$, $P<0.001$, respectively). GLUT-1 AS-ODNs combined with HIF-1 $\alpha$ AS-ODNs, GLUT-1 AS-ODNs combined with 10 Gy X-ray irradiation, and HIF- $1 \alpha$ AS-ODNs combined with 10 Gy X-ray irradiation had synergistic interaction effects on decreasing MVD in Hep-2 cells 8 days after treatment $(P=0.015,0.002,0.026$, respectively) (Table 1) (Table 2).

Cell membrane rupture, cell loss, nuclear concentration, nuclear fragmentation and nuclear dissolution were considered as tumor cells necrosis (Figure 7) (Figure 8). There was a synergistic interaction 
effect of the three treatments combined on decreasing necrosis in Hep-2 and Tu212 xenografts 8 days after treatment $(P<0.001, P=0.004$, respectively), and in Hep2 and Tu212 xenografts 12 days after treatment $(P<0.001$, $P=0.041$, respectively) (Table 1) (Table 2).

It indicated that the formulation, GLUT-1 AS-ODNs $(100 \mu \mathrm{g})$ and HIF-1 $\alpha$ AS-ODNs $(100 \mu \mathrm{g})$ and 10Gy X-ray irradiation are optimized formulations with higher AI, higher necrosis rate and lower MVD of xenografts.

\section{Correlations among GLUT-1 expression, HIF-1 $\alpha$ expression and SUVmax $(\mathrm{T} / \mathrm{N})$}

GLUT1 and HIF-1 $\alpha$ mRNA expression revealed a significant correlation (Pearson's analysis) 8 days ( $R=0.69, p=0.001$ in Hep-2 xenografts, $R=0.639$, $p=0.001$ in Tu212 xenografts)and 12 days after treatment ( $R=0.672, p=0.001$ in Hep-2 xenografts, $R=0.599$, $p=0.002$ in Tu212 xenografts) . GLUT1 and HIF-1 $\alpha$ protein levels revealed a significant correlation (Pearson's analysis) 8 days ( $R=0.63, p=0.001$ in Hep-2 xenografts, $R=0.772, p=0.001$ in Tu212 xenografts) and 12 days after treatment $(R=0.691, p=0.001$ in Hep- 2 xenografts, $R=0.73, p=0.001$ in Tu212 xenografts) .

The tumors grew in the right flanks of the mice, micro PET imaging of tumor xenografts demonstrated ${ }^{18}$ F-FDG uptake. However, GLUT1 and HIF-1 $\alpha$ mRNA and protein expression showed no statistical correlations with SUVmaxT/N2 in both Hep-2 and Tu212 xenografts.

\section{Correlations between ${ }^{18}$ F-FDG accumulation and therapeutic effects}

SUVmaxT/N was used to represent ${ }^{18} \mathrm{~F}-\mathrm{FDG}$ accumulation. necrosis and apoptosis were considered to represent the therapeutic effect. We found that in both Hep-2 and Tu212 xenografts, SUVmaxT/N0 show no statistical significant difference between the groups.

B
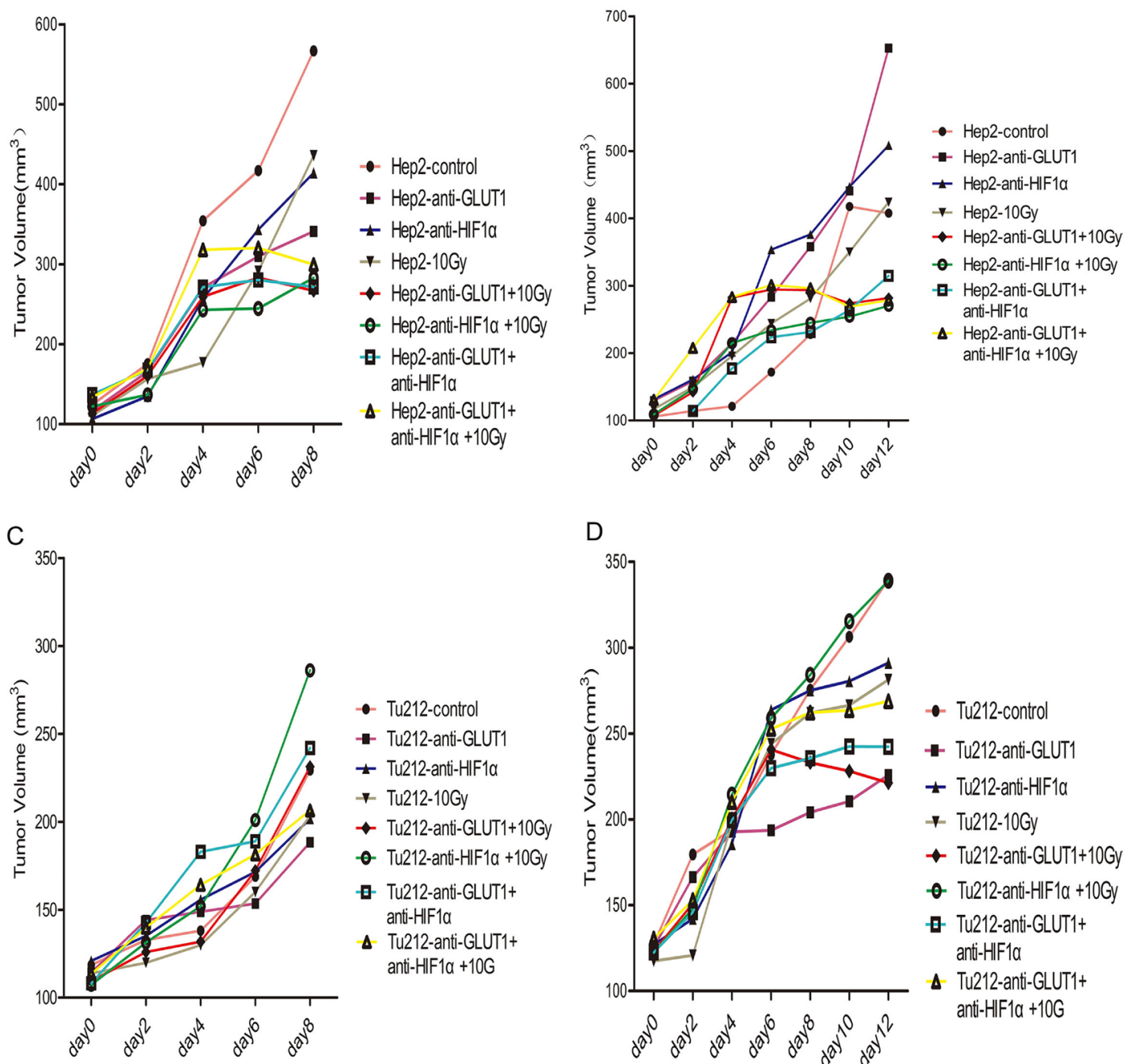

Figure 1: Tumor volumes were calculated every 2 days. The volumes of Hep-2 xenografts 8 days after treatment (A), Hep-2 xenografts 12 days after treatment (B), and Tu212 xenografts 8 days after treatment (C) and Tu212 xenografts 12 days after treatment (D). 
Table 3: Observed GLUT-1 and HIF-1 $\alpha$ expressions of xenografts 8 days after treatment in the $2^{3}$ factorial design with three independent parameters and their two levels (GLUT-1 AS-ODNs and HIF-1 $\alpha$ AS-ODNs $100 \mu \mathrm{g}$ or $0 \mu \mathrm{g}$, X-ray irradiation 10 Gy or 0 Gy)

\begin{tabular}{|c|c|c|c|c|c|c|c|c|c|c|c|}
\hline \multirow{2}{*}{ G } & \multirow{2}{*}{$\mathrm{A}(\mu \mathrm{g})$} & \multirow{2}{*}{ B $(\mu \mathrm{g})$} & \multirow{2}{*}{$\mathrm{C}(\mathrm{Gy})$} & \multicolumn{2}{|c|}{ GLUT-1mRNA $^{\mathrm{x}}$} & \multicolumn{2}{|c|}{ Glut-1 protein ${ }^{\mathrm{X}}$} & \multicolumn{2}{|c|}{ HIF-1 $\alpha$ mRNA $^{x}$} & \multicolumn{2}{|c|}{ HIF-1 $\alpha \operatorname{protein}^{\mathrm{x}}$} \\
\hline & & & & Hep-2 & Tu212 & Нер-2 & Tu212 & Hep-2 & Tu212 & Нер-2 & Tu212 \\
\hline G1 & 100 & 100 & 10 & $0.15 \pm 0.01$ & $0.02 \pm 0.01$ & $0.32 \pm 0.10$ & $0.07 \pm 0.03$ & $0.36 \pm 0.02$ & $0.25 \pm 0.03$ & $0.36 \pm 0.06$ & $0.03 \pm 0.00$ \\
\hline G2 & 100 & 100 & 0 & $0.13 \pm 0.01$ & $0.03 \pm 0.01$ & $0.22 \pm 0.11$ & $0.07 \pm 0.02$ & $0.45 \pm 0.05$ & $0.40 \pm 0.07$ & $0.10 \pm 0.05$ & $0.06 \pm 0.03$ \\
\hline G3 & 0 & 100 & 10 & $0.71 \pm 0.05$ & $0.26 \pm 0.03$ & $0.85 \pm 0.14$ & $0.20 \pm 0.09$ & $0.45 \pm 0.04$ & $0.25 \pm 0.05$ & $0.60 \pm 0.21$ & $0.07 \pm 0.03$ \\
\hline G4 & 100 & 0 & 10 & $0.91 \pm 0.08$ & $0.12 \pm 0.01$ & $0.81 \pm 0.20$ & $0.09 \pm 0.02$ & $1.10 \pm 0.09$ & $0.59 \pm 0.02$ & $0.91 \pm 0.08$ & $0.11 \pm 0.02$ \\
\hline G5 & 0 & 0 & 10 & $1.09 \pm 0.09$ & $0.49 \pm 0.07$ & $0.67 \pm 0.21$ & $0.45 \pm 0.03$ & $1.18 \pm 0.16$ & $0.67 \pm 0.05$ & $0.63 \pm 0.12$ & $0.22 \pm 0.04$ \\
\hline G6 & 0 & 100 & 0 & $0.34 \pm 0.02$ & $0.22 \pm 0.05$ & $0.14 \pm 0.09$ & $0.22 \pm 0.06$ & $0.27 \pm 0.01$ & $0.34 \pm 0.01$ & $0.13 \pm 0.02$ & $0.10 \pm 0.07$ \\
\hline G7 & 100 & 0 & 0 & $0.20 \pm 0.02$ & $0.11 \pm 0.02$ & $0.37 \pm 0.06$ & $0.11 \pm 0.03$ & $0.95 \pm 0.06$ & $0.92 \pm 0.04$ & $0.63 \pm 0.14$ & $0.17 \pm 0.09$ \\
\hline G8 & 0 & 0 & 0 & $1.00 \pm 0.08$ & $1.02 \pm 0.15$ & $1.07 \pm 0.07$ & $0.84 \pm 0.09$ & $1.01 \pm 0.07$ & $1.02 \pm 0.14$ & $0.86 \pm 0.07$ & $0.29 \pm 0.06$ \\
\hline \multicolumn{4}{|c|}{ a ( $p$-value $)$} & $<0.001$ & $<0.001$ & 0.018 & $<0.001$ & 0.639 & 0.510 & 0.509 & 0.121 \\
\hline \multicolumn{4}{|c|}{$\mathrm{b}$ ( $p$-value) } & $<0.001$ & $<0.001$ & 0.002 & $<0.001$ & $<0.001$ & $<0.001$ & $<0.001$ & 0.003 \\
\hline \multicolumn{4}{|c|}{$\mathrm{c}$ ( $p$-value) } & $<0.001$ & 0.012 & 0.043 & 0.014 & 0.127 & $<0.001$ & 0.028 & 0.326 \\
\hline \multicolumn{4}{|c|}{$\mathrm{ab}$ ( $p$-value) } & 0.128 & $<0.001$ & 0.789 & $<0.001$ & 0.238 & 0.212 & 0.331 & 0.547 \\
\hline \multicolumn{4}{|c|}{ ac ( $p$-value) } & 0.092 & 0.013 & 0.567 & 0.025 & 0.275 & 0.755 & 0.382 & 0.783 \\
\hline \multicolumn{4}{|c|}{ bc ( $p$-value) } & 0.048 & 0.007 & 0.063 & 0.03 & 0.401 & 0.026 & 0.051 & 0.750 \\
\hline \multicolumn{4}{|c|}{ abc ( $p$-value) } & $<0.001$ & $<0.01$ & 0.002 & 0.041 & 0.202 & 0.680 & 0.002 & 0.805 \\
\hline
\end{tabular}

G: group, A: GLUT-1 AS-ODNs, B: HIF-1 $\alpha$ AS-ODNs, C: X-ray irradiation.

a: main effect of GLUT-1 AS-ONDs, b: main effect of HIF-1 $\alpha$ AS-ODNs, c: main effect of X-ray irradiation, ab: interaction effect between GLUT-1 AS-ONDs and HIF-1 $\alpha$ ASODNs, ac: interaction effect between GLUT-1 AS-ONDs and X-ray irradiation, bc: interaction effect between HIF-1 $\alpha$ AS-ODNs and X-ray irradiation, abc: interaction effect between GLUT-1 AS-ONDs and HIF-1 $\alpha$ AS-ODNs and X-ray irradiation.

${ }^{\mathrm{x}}$ Mean $\pm \operatorname{Std}($ standard deviation).

Table 4: Observed GLUT-1 and HIF-1 $\alpha$ expressions of xenografts 12 days after treatment in the $2^{3}$ factorial design with three independent parameters and their two levels (GLUT-1 AS-ODNs and HIF-1 $\alpha$ AS-ODNs $100 \mu$ or $0 \mu \mathrm{g}$, X-ray irradiation 10 Gy or 0 Gy)

\begin{tabular}{|c|c|c|c|c|c|c|c|c|c|c|c|}
\hline \multicolumn{12}{|c|}{ The results detected 12 days after treatment of xenografts } \\
\hline \multirow{2}{*}{ G } & \multirow{2}{*}{$\begin{array}{c}\mathrm{A} \\
(\mu \mathrm{g})\end{array}$} & \multirow{2}{*}{$\begin{array}{c}\text { B } \\
(\mu \mathrm{g})\end{array}$} & \multirow{2}{*}{$\begin{array}{c}\text { C } \\
\text { (Gy) }\end{array}$} & \multicolumn{2}{|c|}{ GLUT-1mRNA $^{X}$} & \multicolumn{2}{|c|}{ Glut-1 protein $^{x}$} & \multicolumn{2}{|c|}{ HIF-1 $\alpha$ mRNA ${ }^{X}$} & \multicolumn{2}{|c|}{ HIF-1 $\alpha$ protein $^{\mathrm{x}}$} \\
\hline & & & & Нер-2 & Tu212 & Нер-2 & Tu212 & Нер-2 & Tu212 & Hep-2 & Tu212 \\
\hline G1 & 100 & 100 & 10 & $0.51 \pm 0.02$ & $0.20 \pm 0.04$ & $0.54 \pm 0.08$ & $0.04 \pm 0.01$ & $0.14 \pm 0.01$ & $0.26 \pm 0.03$ & $0.07 \pm 0.02$ & $0.03 \pm 0.00$ \\
\hline $\mathrm{G} 2$ & 100 & 100 & 0 & $0.62 \pm 0.03$ & $0.36 \pm 0.02$ & $0.82 \pm 0.01$ & $0.17 \pm 0.02$ & $0.31 \pm 0.01$ & $0.38 \pm 0.01$ & $0.19 \pm 0.08$ & $0.05 \pm 0.01$ \\
\hline G3 & 0 & 100 & 10 & $0.33 \pm 0.02$ & $0.59 \pm 0.09$ & $0.55 \pm 0.15$ & $0.26 \pm 0.06$ & $0.40 \pm 0.03$ & $0.34 \pm 0.04$ & $0.18 \pm 0.06$ & $0.09 \pm 0.03$ \\
\hline G4 & 100 & 0 & 10 & $0.50 \pm 0.05$ & $0.31 \pm 0.04$ & $0.76 \pm 0.02$ & $0.13 \pm 0.02$ & $0.34 \pm 0.03$ & $0.59 \pm 0.08$ & $0.32 \pm 0.02$ & $0.32 \pm 0.13$ \\
\hline G5 & 0 & 0 & 10 & $0.80 \pm 0.06$ & $0.44 \pm 0.06$ & $0.59 \pm 0.18$ & $0.57 \pm 0.15$ & $0.65 \pm 0.08$ & $0.54 \pm 0.01$ & $0.42 \pm 0.08$ & $0.29 \pm 0.07$ \\
\hline G6 & 0 & 100 & 0 & $0.31 \pm 0.03$ & $0.50 \pm 0.04$ & $0.59 \pm 0.12$ & $0.48 \pm 0.08$ & $0.21 \pm 0.02$ & $0.50 \pm 0.02$ & $0.28 \pm 0.08$ & $0.12 \pm 0.00$ \\
\hline G7 & 100 & 0 & 0 & $0.78 \pm 0.05$ & $0.48 \pm 0.11$ & $0.94 \pm 0.10$ & $0.39 \pm 0.11$ & $0.83 \pm 0.04$ & $1.01 \pm 0.12$ & $0.64 \pm 0.15$ & $0.29 \pm 0.05$ \\
\hline \multirow[t]{8}{*}{ G8 } & 0 & 0 & 0 & $1.00 \pm 0.07$ & $1.01 \pm 0.11$ & $1.10 \pm 0.20$ & $1.40 \pm 0.39$ & $1.00 \pm 0.05$ & $1.01 \pm 0.11$ & $0.66 \pm 0.04$ & $0.54 \pm 0.15$ \\
\hline & \multicolumn{2}{|c|}{ a ( $p$-value) } & & 0.023 & $<0.001$ & 0.506 & 0.001 & $<0.001$ & 0.515 & 0.289 & 0.142 \\
\hline & \multicolumn{2}{|c|}{$\mathrm{b}$ ( $p$-value) } & & $<0.001$ & 0.01 & 0.036 & 0.004 & $<0.001$ & $<0.001$ & $<0.001$ & 0.001 \\
\hline & \multicolumn{2}{|c|}{ c ( $p$-value) } & & 0.02 & 0.001 & 0.017 & 0.006 & $<0.001$ & $<0.001$ & 0.014 & 0.006 \\
\hline & \multicolumn{2}{|c|}{$\mathrm{ab}$ ( $p$-value) } & & $<0.001$ & 0.049 & 0.618 & 0.058 & 0.021 & 0.200 & 0.754 & 0.69 \\
\hline & \multicolumn{2}{|c|}{ ac ( $p$-value) } & & 0.011 & 0.05 & 0.874 & 0.1570 & 0.001 & 0.623 & 0.727 & 0.217 \\
\hline & \multicolumn{2}{|c|}{ bc ( $p$-value) } & & 0.001 & 0.006 & 0.386 & 0.1221 & $<0.001$ & 0.004 & 0.244 & 0.473 \\
\hline & \multicolumn{2}{|c|}{ abc ( $p$-value) } & & 0.001 & $<0.01$ & 0.164 & 0.305 & 0.107 & 0.936 & 0.793 & 0.228 \\
\hline
\end{tabular}

G: group, A: GLUT-1 AS-ODNs, B: HIF-1 $\alpha$ AS-ODNs,C: X-ray irradiation.

a: main effect of GLUT-1 AS-ONDs, $b$ : main effect of HIF-1 $\alpha$ AS-ODNs, c: main effect of X-ray irradiation, ab: interaction effect between GLUT-1 AS-ONDs and HIF-1 $\alpha$ ASODNs, ac: interaction effect between GLUT-1 AS-ONDs and X-ray irradiation, bc: interaction effect between HIF-1 $\alpha$ AS-ODNs and X-ray irradiation, abc: interaction effect between GLUT-1 AS-ONDs and HIF-1 $\alpha$ AS-ODNs and X-ray irradiation.

${ }^{\mathrm{x}} \mathrm{Mean} \pm \operatorname{Std}($ standard deviation). 

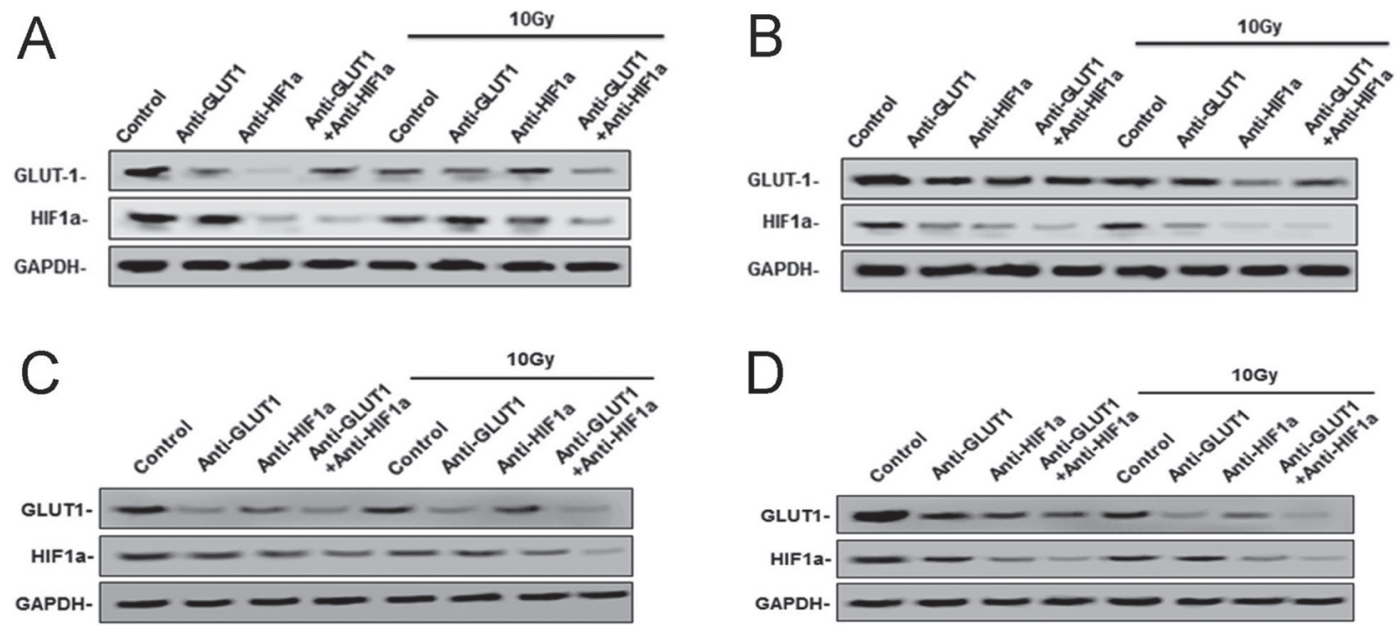

Figure 2: West-blotting analysis showed the protein levels of GLUT-1 and HIF-1a protein. The expression of GLUT-1, HIF-1 $\alpha$ protein in Hep-2 xenografts 8 days after treatment (A), 12 days after treatment (B) and Tu212 xenografts 8 days after treatment (C) and 12 days after treatment (D).
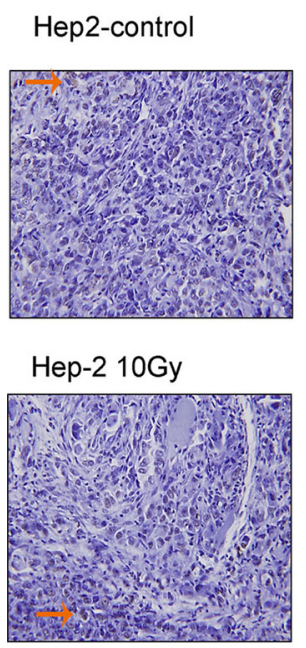

Tu212-control
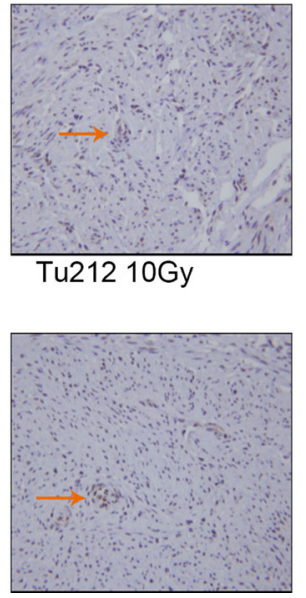

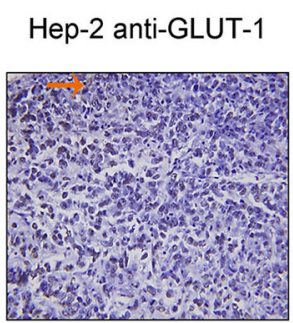

Hep-2 anti-GLUT-1+ $10 \mathrm{~Gy}$

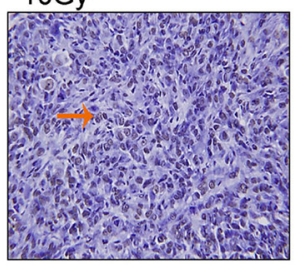

Tu212 anti-GLUT-1
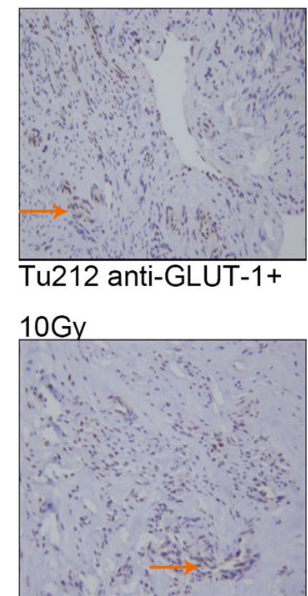

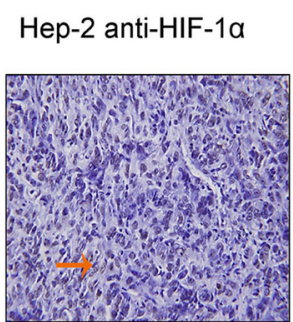

Hep-2 anti-HIF-1a+

$10 \mathrm{~Gy}$

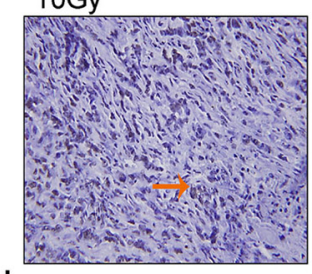

Tu212 anti-HIF-1a
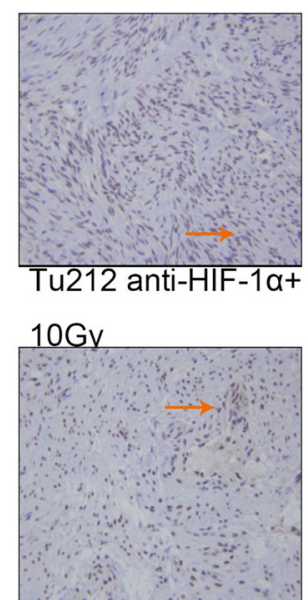

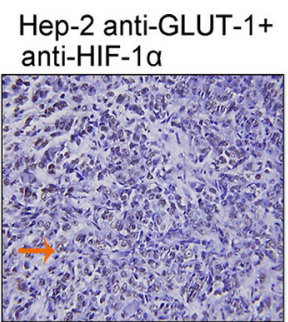

Hep-2 anti-GLUT-1+ anti-HIF-1a+10Gy

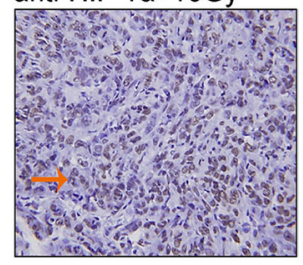

Tu212 anti-GLUT-1+ anti-HIF-1a
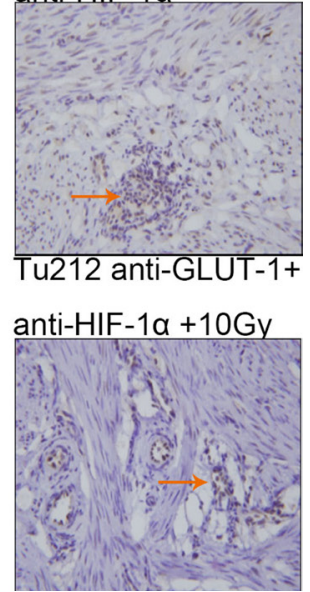

Figure 3: TUNEL positive staining showed that the nucleus was brown or brown-yellow, that is, apoptotic cells. The arrows in the figure refer to apoptotic cells of Hep-2 and Tu212 xenografts 8 days after treatment. Apoptosis index were observed under an optical microscope (magnification, $\times 400$ ). 
Neither SUVmaxT/N1, SUVmaxT/N2, $\Delta$ SUVmaxT/N1, $\triangle \mathrm{SUVmaxT} / \mathrm{N} 2$ nor $\Delta \mathrm{SUVmaxT} / \mathrm{N} 12$ show a statistical correlation with therapeutic effect (necrosis and apoptosis) in Hep-2 or Tu212 xenografts (Figure 9). Thus, we found no value in using SUVmaxT/N to evaluate therapeutic effects.

\section{DISCUSSION}

Inhibiting the expression of HIF-1 $\alpha$ to promote tumor radiosensitivity has been demonstrated in various preclinical studies [27, 28]. Tumor glucose metabolism can be targeted either directly by inhibiting enzymes and transporters involved in glucose metabolism or indirectly by anti-HIF-1 therapy [29]. In this study, we found GLUT-1 expression in the xenografts was inhibited significantly by
HIF-1 $\alpha$ AS-ODNs. HIF-1 $\alpha$ AS-ODNs could decreased the expression of GLUT-1 mRNA and protein. It was consistent with other studies. In the study of Chen et al, they found inhibiting the expression of HIF-1 $\alpha$ could decrease the expression level of GLUT-1, and thereby inhibited the volume and tumor weight of LOVO cell line xenografts [30]. Fan et al found that lentiviral vector-mediated RNA interference targeting HIF-1 $\alpha$ significantly inhibited the expression of Glut-1 mRNA in Patu8988 pancreatic cancer cells [31]. In our study, GLUT-1 AS-ODNs alone didn't reduce the GLUT-1 protein levels in Hep-2 xenografts 12 days after treatment, however, in Tu212 xenografts 8 days and 12 days after treatment and in Hep-2 xenografts 8 days after treatment, GLUT-1 AS-ODNs alone decreased GLUT-1 protein expression significantly. This difference might be induced by changes in the stabilities of GLUT-1 mRNA.
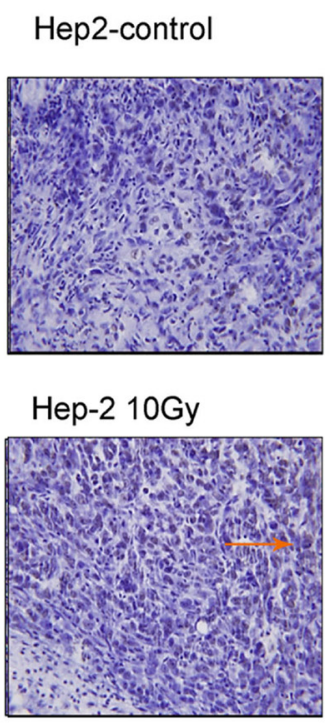

Tu212-control

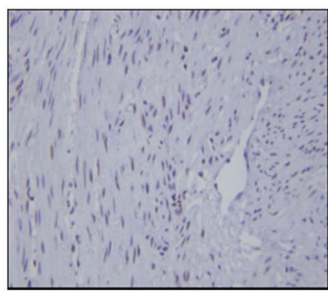

Tu212 10Gy

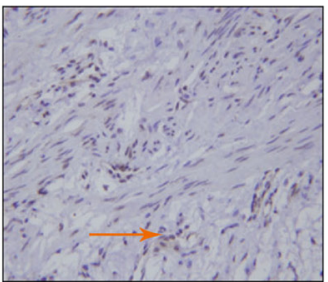

Hep-2 anti-GLUT-1

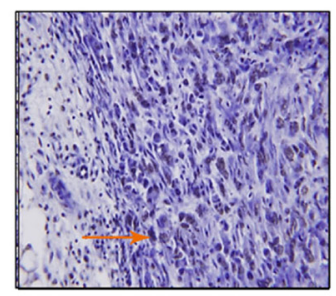

Hep-2 anti-GLUT-1+ $10 \mathrm{~Gy}$

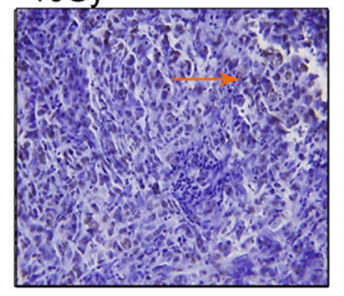

Tu212 anti-GLUT-1

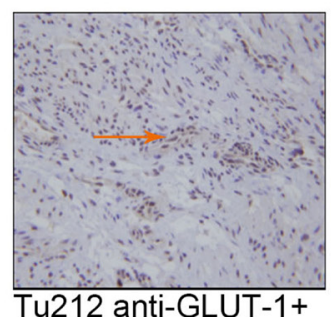

$10 G y$

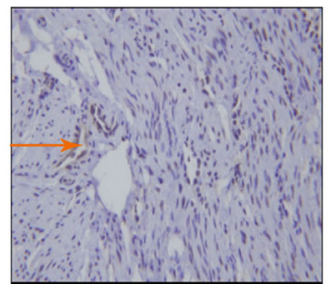

Hep-2 anti-HIF-1a

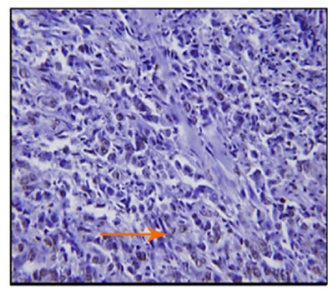

Hep-2 anti-HIF-1a+ $10 \mathrm{~Gy}$

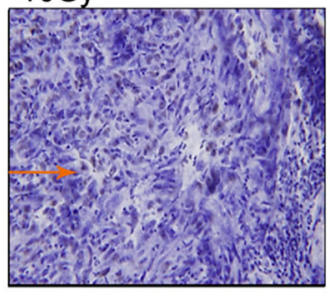

Tu212 anti-HIF-1a

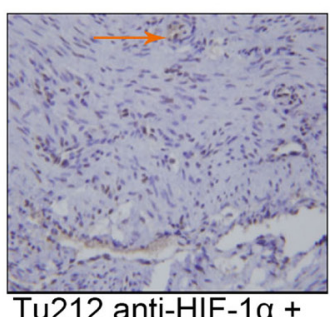

$10 \mathrm{~Gy}$

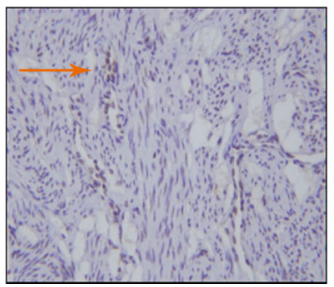

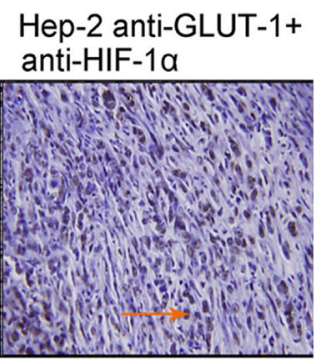

Hep-2 anti-GLUT-1+

anti-HIF-1a+10Gy

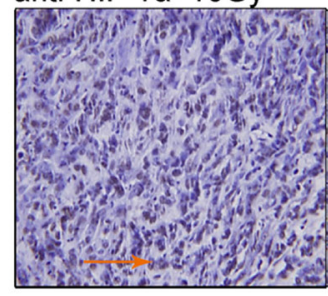

Tu212 anti-GLUT-1+

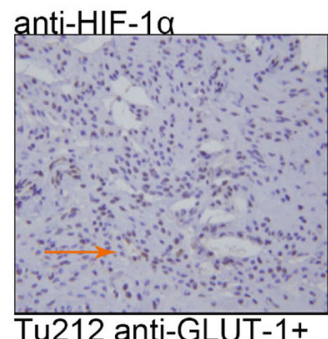

anti-HIF-1 $\alpha+10$ Gy

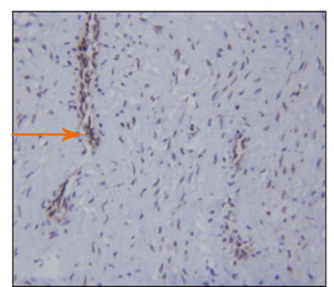

Figure 4: Apoptotic cells of Hep-2 and Tu212 xenografts 12 days after treatment pointed out with arrows. Apoptosis index were observed under an optical microscope (magnification, $\times 400$ ). 
Interfering with glucose metabolism in cancer cells to reduce levels of antioxidant metabolites could thus promote radiosensitivity [32]. Meijer et al found that targeting HIF-1 and glucose metabolism impacted the tumor microenvironment, and sensitized various solid tumors to irradiation [32].

Our data indicated a synergistic interaction effect among the three treatments on increasing apoptosis in Hep-2 and Tu212 xenografts. Some studies have shown that inhibiting the expression of HIF-1 $\alpha$ or GLUT-1 alone increased tumor cell apoptosis [33-36]. Our results presented here showed that simultaneous inhibition of GLUT-1 and HIF-1 $\alpha$ promoted tumor cell apoptosis more effectively than did inhibition of GLUT-1 or HIF-1 $\alpha$ alone.

Angiogenesis could stimulate malignant tumor occurrence, development, invasion and metastasis.
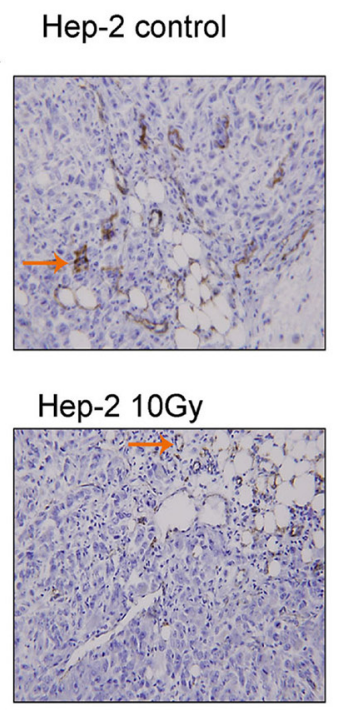

Tu212 control
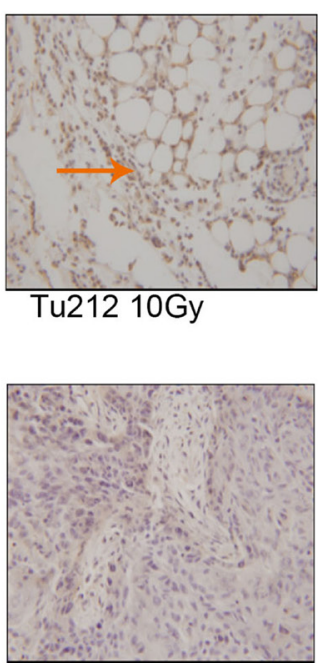

Hep-2 anti-GLUT-1
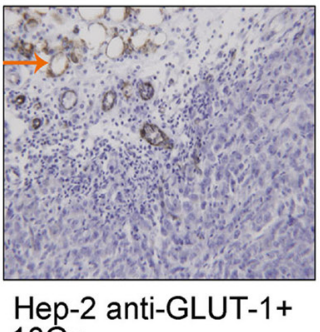

$10 \mathrm{~Gy}$

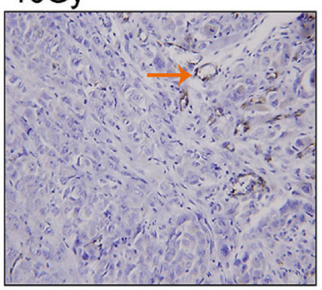

Tu212 anti-GLUT-1

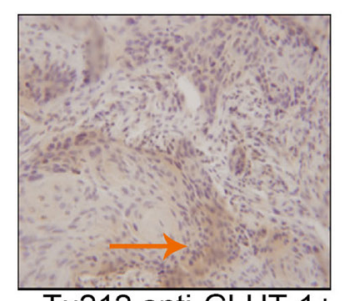

Tu212 anti-GLUT-1+

$+10 \mathrm{~Gy}$

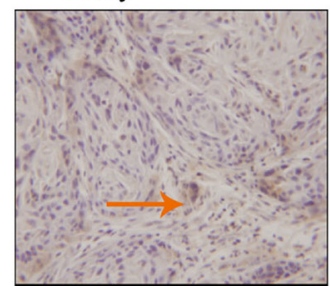

However, there have been no reports regarding the role of combined inhibition of HIF- $1 \alpha$ and GLUT-1 on angiogenesis in laryngeal squamous carcinoma. In this study, there was a synergistic interaction effect of the three treatments on decreasing MVD in Hep-2 and Tu212 xenografts. Some studies have shown that downregulation of HIF-1 $\alpha$ alone decreased intratumoral MVD [37, 38]. However, others have found that HIF-1 $\alpha$ and GLUT-1 were not correlated with MVD in patients with locally advanced cervical cancer [39]. In the present study, the results showed that simultaneous inhibition of GLUT-1 and HIF- $1 \alpha$ decreased MVD more effectively than did inhibition of GLUT-1 or HIF- $1 \alpha$ alone in laryngeal cancer.

A synergistic interaction effect of the three treatments on increasing necrosis was also seen in Hep-2 and $\mathrm{Tu} 212$ xenografts. Some studies have demonstrated
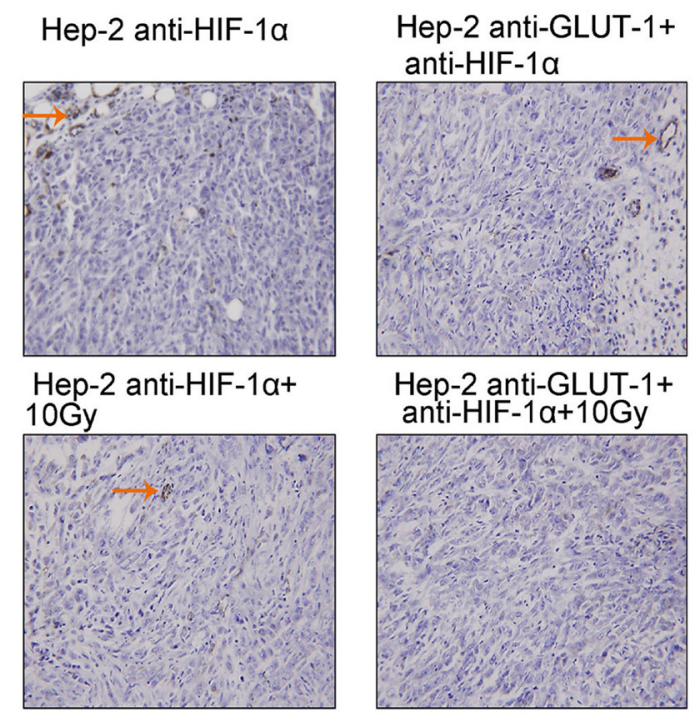

Tu212 anti-HIF-1a

Tu212 anti-GLUT-1+
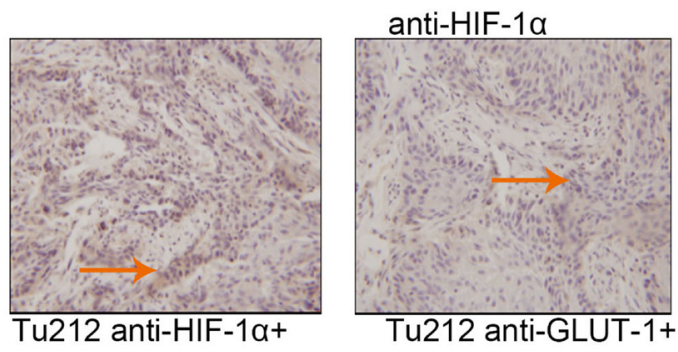

$10 \mathrm{~Gy}$

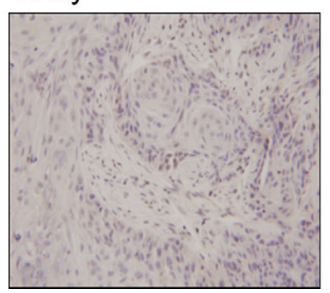

anti-HIF-1 $\alpha+10 G y$

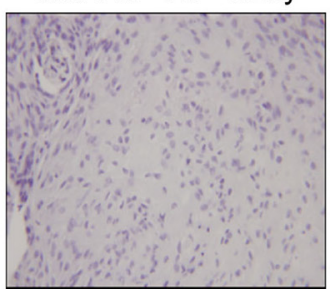

Figure 5: CD34 is the most sensitive marker of vascular endothelial cells, positive staining showed cytoplasm was light brown to brown. The arrows in the figure refer to apoptotic cells of Hep- 2 and Tu2 12 xenografts 8 days after treatment. Microvessel density were observed under an optical microscope (magnification, $\times 200$ ). 
that inhibition of HIF-1 or GLUT-1 alone increased tumor cell necrosis $[40,41]$. However, Petty et al found no significant correlation between GLUT-1 and the percentage of necrosis in canine osteosarcoma [42]. In the present study, the results showed that simultaneous inhibition of GLUT-1 and HIF-1 $\alpha$ increased necrosis more effectively than did inhibition of GLUT-1 or HIF-1 $\alpha$ alone in laryngeal cancer.

Although GLUT-1 AS-ODNs and HIF-1 $\alpha$ AS-ODNs did not influence the weight or volume of the xenografts, they did increase tumor AI, increase tumor necrosis and

Hep-2 control
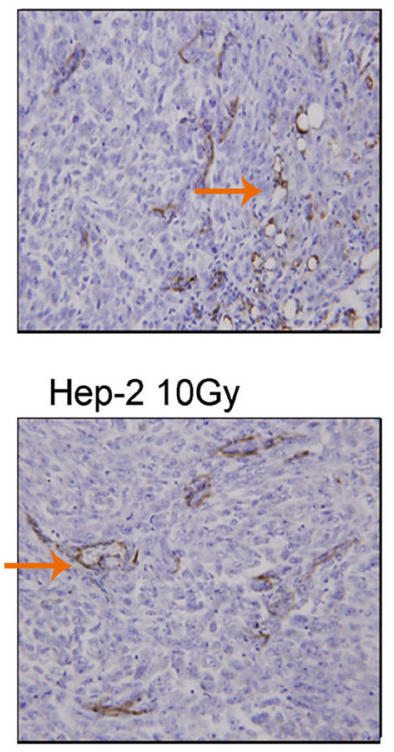

Tu212 control

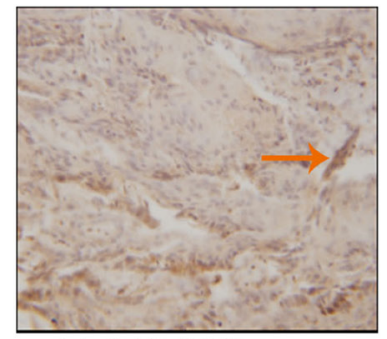

Tu212 10Gy

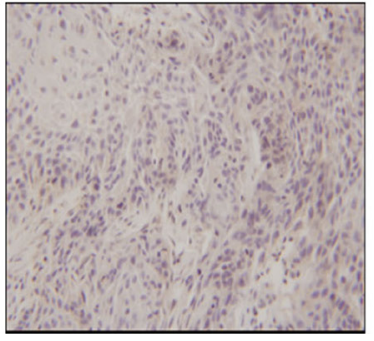

Hep-2 anti-GLUT-1
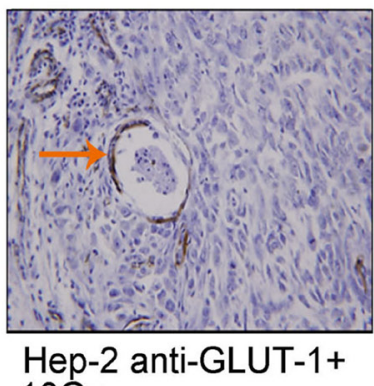
$10 \mathrm{~Gy}$

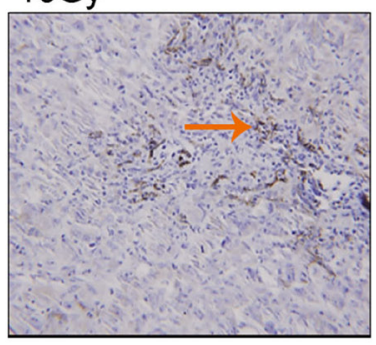

Tu212 anti-GLUT-1
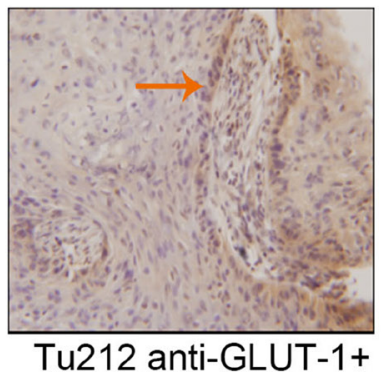
$10 \mathrm{~Gy}$

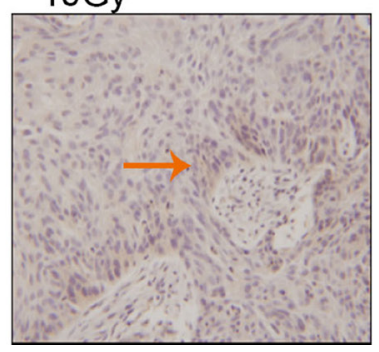

decrease tumor MVD. GLUT-1 AS-ODNs and HIF-1 $\alpha$ AS-ODNs might increase the radiosensitivity of Hep-2 and Tu212 xenografts.

In our experiments, GLUT1 and HIF-1 $1 \alpha$ mRNA, and protein levels showed significant linear correlations, however, neither GLUT1 nor HIF-1 $\alpha$ expression was correlated with SUVmaxT/N. Our results were similar to those of other studies in which ${ }^{18} \mathrm{~F}$-FDG uptake did not correlate with GLUT-1 [43, 44], or HIF-1 $\alpha$ expression [45]. However, other studies demonstrated that ${ }^{18} \mathrm{~F}$-FDG uptake was correlated with HIF-1 $\alpha$ [46],

Hep-2 anti-HIF-1 $\alpha$
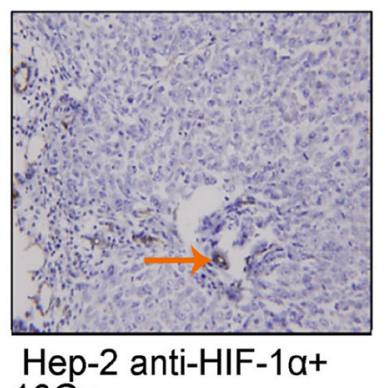

10Gy

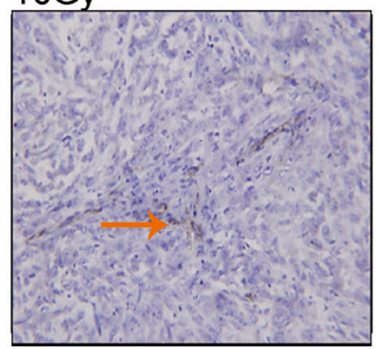

Tu212 anti-HIF-1a
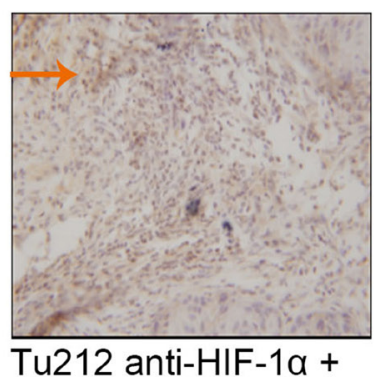

10Gy

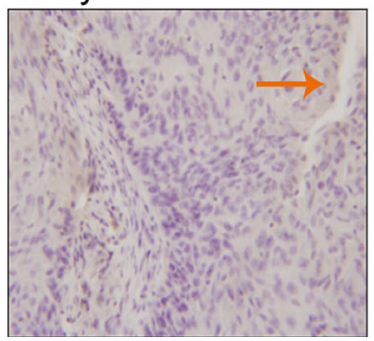

Hep-2 anti-GLUT-1+ anti-HIF-1a

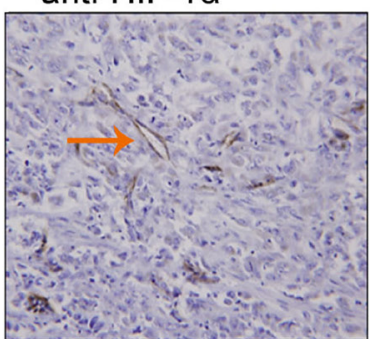

Hep-2 anti-GLUT-1+ anti-HIF-1a+10Gy

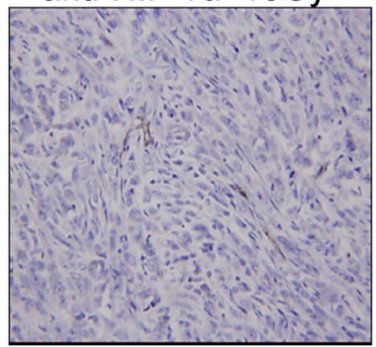

Tu212 anti-GLUT-1+

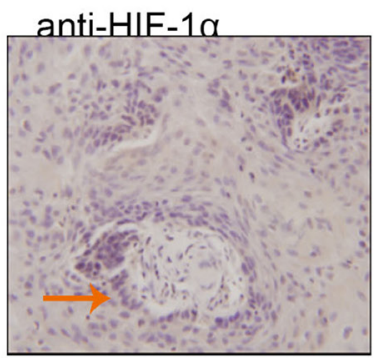

Tu212 anti-GLUT-1+

anti-HIF-1 $\alpha+10 G y$

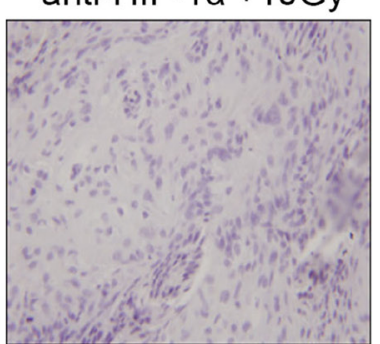

Figure 6: Microvessels of Hep-2 and Tu212 xenografts 12 days after treatment pointed out with arrows. Microvessel density were observed under an optical microscope (magnification, $\times 200$ ). 
and GLUT-1 expression [45]. Yamada et al found that in early stage tumor, FDG uptake was associated with the expression of GLUT-1 and HIF-1, however, there were no correlations in the later-stage tumor [47]. These differences are likely explained by factors such as the accuracy of PET system quality control, image reconstruction algorithm and filtering, body composition, time interval between tracer injection and length of uptake period, plasma glucose level and partial volume effects $[48,49]$.
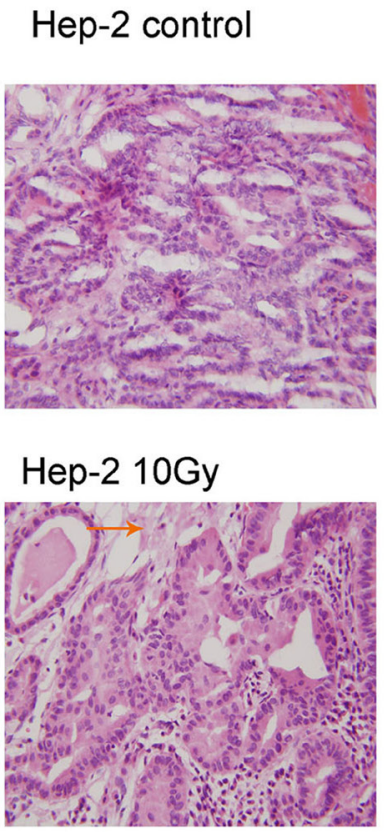

Tu212 control

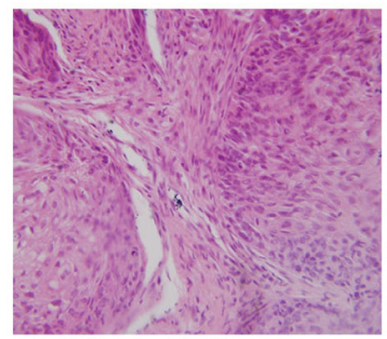

Tu212 10Gy

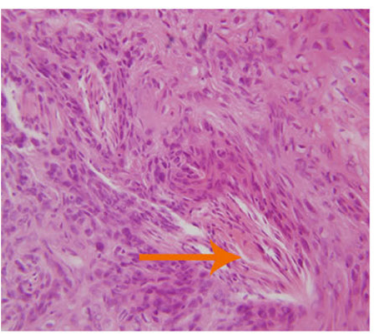

Hep-2 anti-GLUT-1

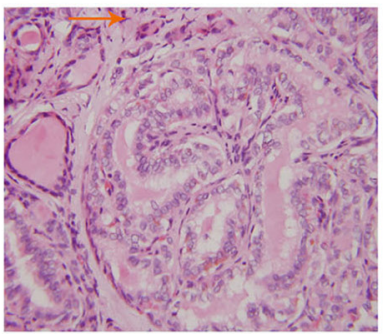

Hep-2 anti-GLUT-1+ $10 \mathrm{~Gy}$

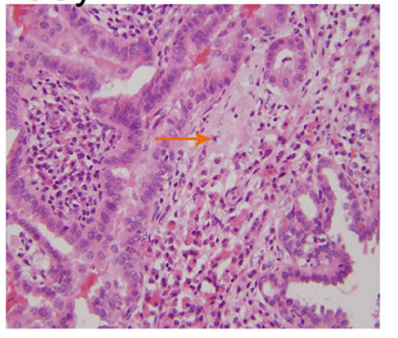

Tu212 anti-GLUT-1
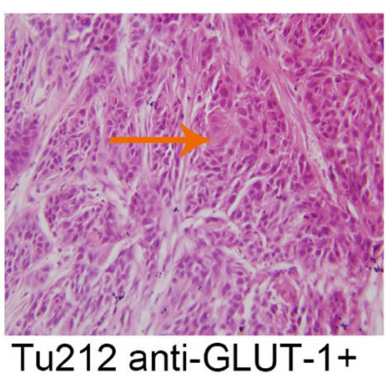

10Gy

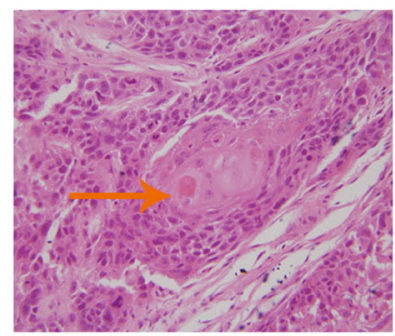

In the present study, neither SUVmaxT/N1, SUVmaxT/N2, $\Delta$ SUVmaxT/N1, $\Delta$ SUVmaxT/N2 nor $\Delta$ SUVmaxT/N12 showed a statistical correlation with therapeutic effects (necrosis, apoptosis). ${ }^{18} \mathrm{~F}-\mathrm{FDG}$ PET for treatment monitoring was also influenced by the varying effectiveness of therapy in different stages of disease and in different tumor types [50]. There is controversy regarding the value of PET/CT in evaluating therapeutic effects on cancers. Some have stated that
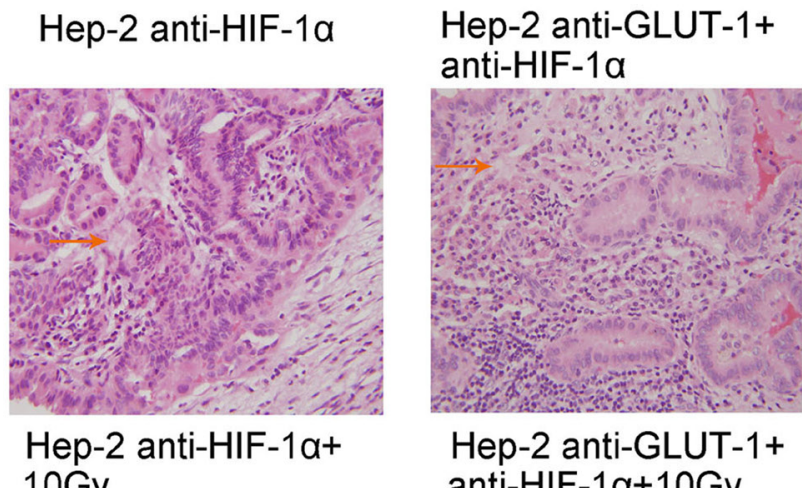

$10 \mathrm{~Gy}$

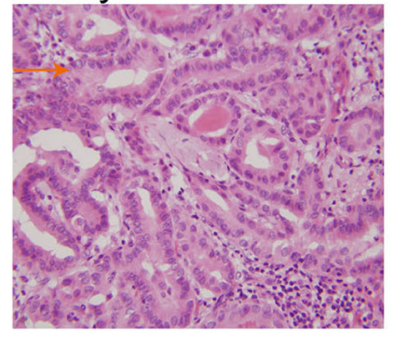

Tu212 anti-HIF-1a

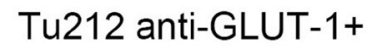

Hep-2 anti-GLUT-1+ anti-HIF-1a+10Gy

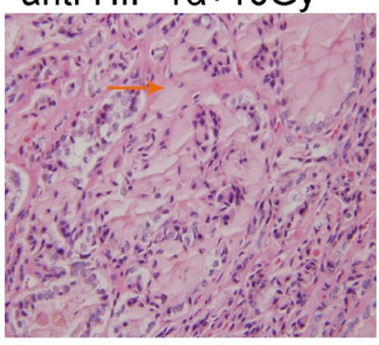

Tu212 anti-GLUT-1+

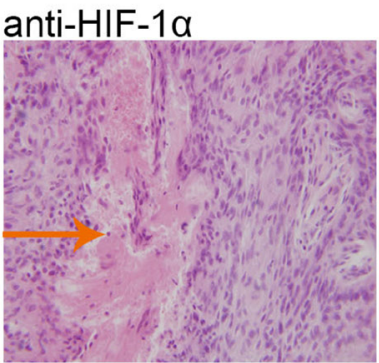

Tu212 anti-GLUT-1+ anti-HIF-1a +10Gy $10 \mathrm{~Gy}$

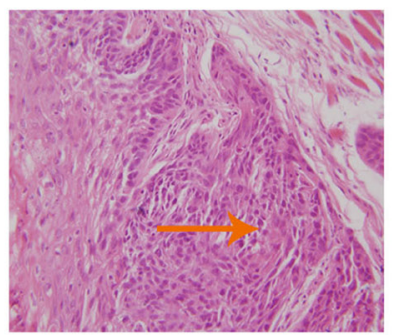

Tu212 anti-HIF-1a +

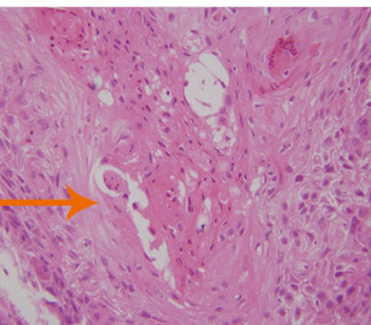

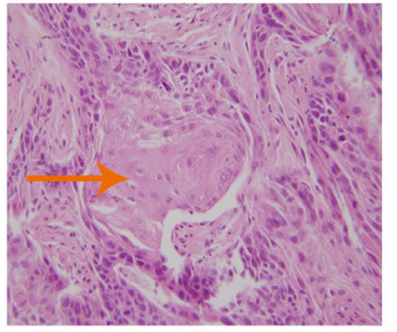

Figure 7: Cell membrane rupture, cell loss, nuclear concentration, nuclear fragmentation and nuclear dissolution were considered as tumor cells necrosis. The arrows in the figure refer to necrosis of Hep-2 and Tu212 xenografts 8 days after treatment. Necrosis were observed under an optical microscope (magnification, $\times 400$ ). 
FDG PET/CT was a valuable tool for assessing treatment responses [21, 51]. ${ }^{18} \mathrm{~F}-\mathrm{FDG}$ uptake was increased in hyperglycolyzed regions, but the exact mechanisms were complex and influenced by several microenvironmental parameters, such as the intracellular ${ }^{18} \mathrm{~F}-\mathrm{FDG}$ phosphorylation capability, tumor oxygenation status, GLUT activity, blood flow and permeability [52-54]. Indeed, in most clinical experiments, FDG PET/CT was found to predict the treatment effect in tumors, however, this was not the case in preclinical experiments involving mouse tumor xenografts.

With respect to in vivo imaging, the proliferative activity of tumor cells has been shown to be more specific
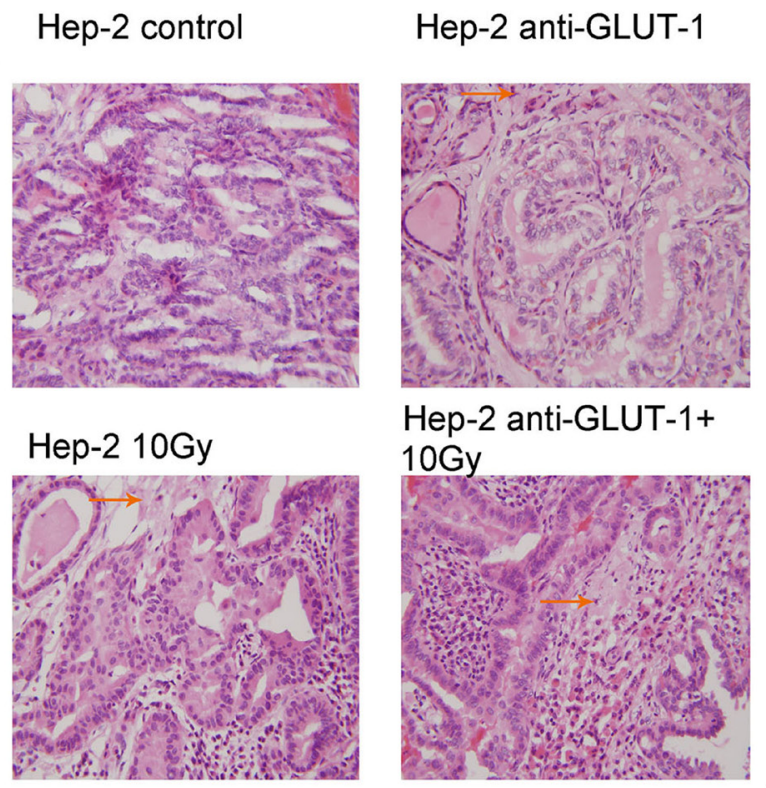

Tu212 control
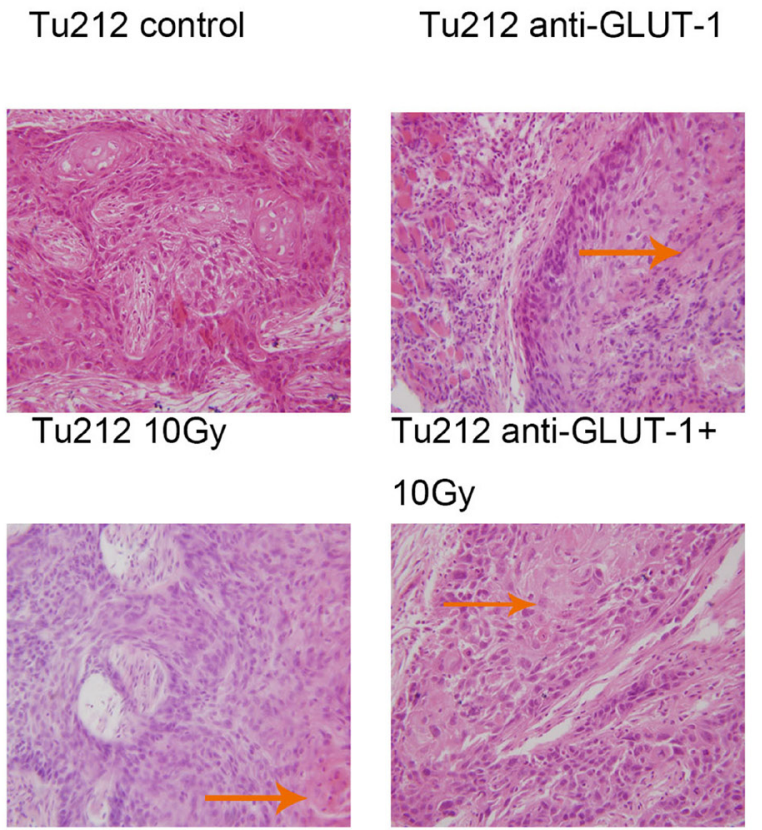

for cancers than has glucose metabolism. Thus, 3'-deoxy$3^{\prime}-\left[{ }^{18} \mathrm{~F}\right]$ fluorothymidine $\left({ }^{18} \mathrm{~F}-\mathrm{FLT}\right)$ may better reflect the proliferative capacity of tumor cells and may be useful in evaluating the therapeutic response [55], as it shows no uptake in inflamed tissues. Compared with ${ }^{18} \mathrm{~F}-\mathrm{FDG}$ uptake in response to irradiation, ${ }^{18} \mathrm{~F}$-FLT coincides more closely with the observed biological responses in cancer cell lines. Thus, in future study, we may use ${ }^{18} \mathrm{~F}$-FLT as a PET tracer to investigate its value in predicting the treatment effect of laryngeal carcinoma.

The present study has several limitations, including the relatively low number of mice used per group, heterogeneity among the mice, the low frequency of drug
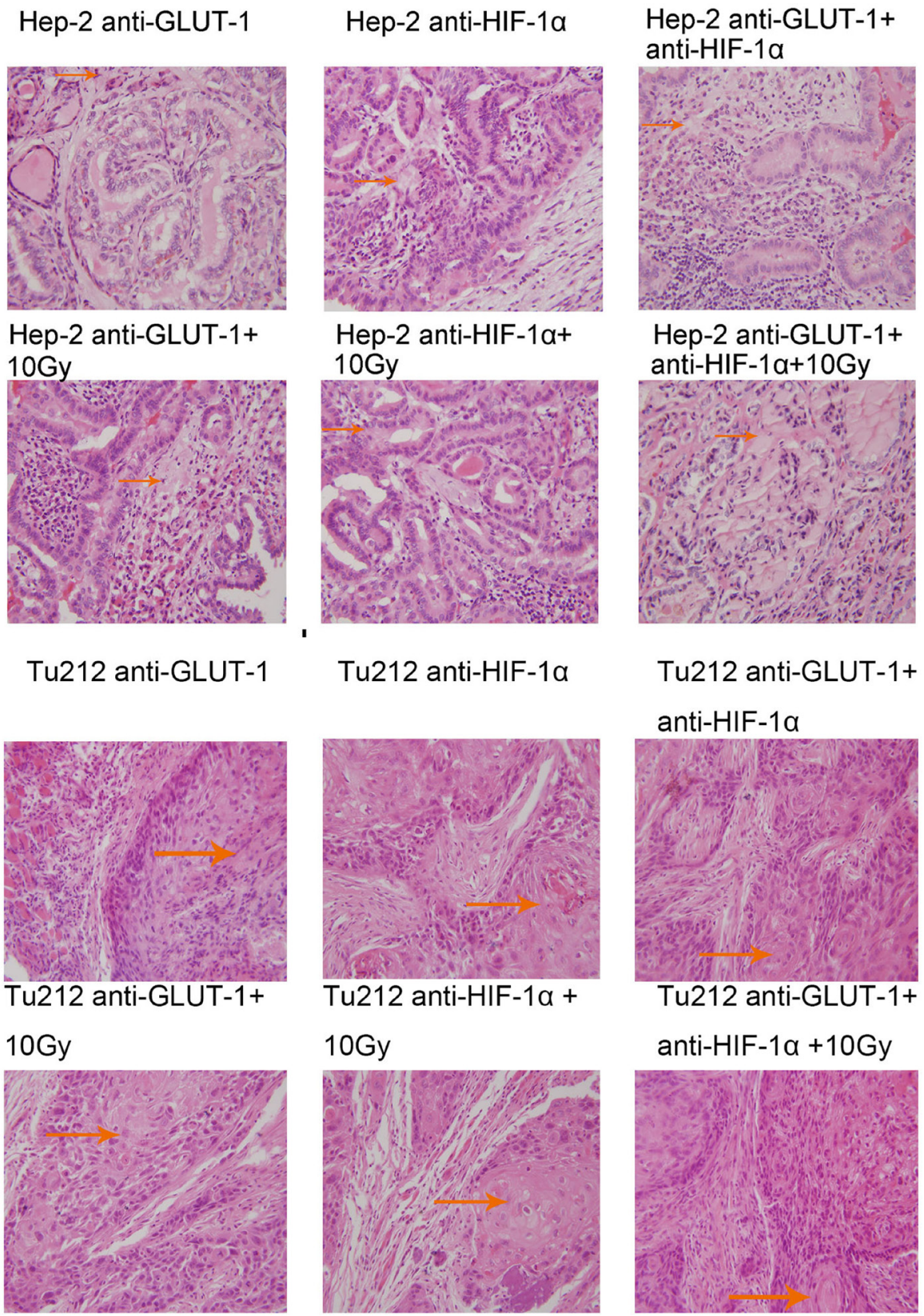

Tu212 anti-GLUT-1+
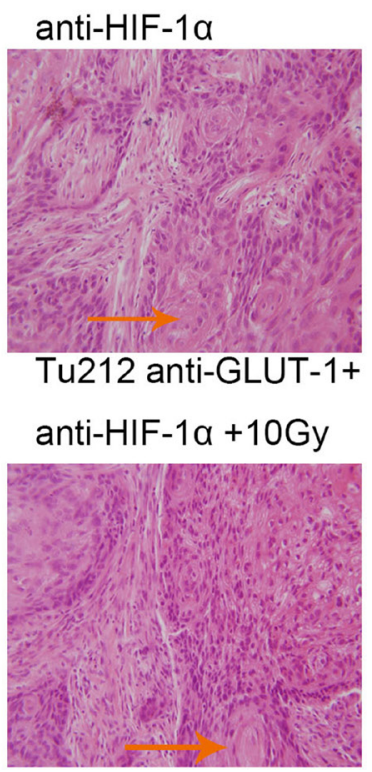

Figure 8: Necrosis of Hep-2 and Tu212 xenografts 12 days after treatment pointed out with arrows. Necrosis were observed under an optical microscope (magnification, $\times 400$ ). 
administration and the influence of environmental factors (e.g air temperature) on PET/CT results. We accept that ${ }^{18} \mathrm{~F}-\mathrm{FDG}$ uptake may also vary depending on tumor characteristics. SUV does not reflect the heterogeneity of a tumor, total lesion glycolysis (TLG) represents both the degree of ${ }^{18} \mathrm{~F}$-FDG uptake and the size of the metabolically active tumor, and TLG has been proposed as a quantitative index of tumor metabolism. Hong et al demonstrated that TLG was a better predictor of survival than was SUV in patients with locally advanced esophageal cancer treated with radiotherapy [56]. Thus, we intend to perform subgroup analyses involving more mice and to evaluate the therapeutic effects on tumor xenografts using TLG in further investigations.

In conclusion, HIF-1 $\alpha$ AS-ODNs and GLUT-1 ASODNs decreased HIF-1 $\alpha$ and GLUT-1 levels, respectively, and HIF-1 $\alpha$ AS-ODNs also decreased GLUT-1 level. Moreover, HIF-1 $\alpha$ AS-ODNs and GLUT-1 AS-ODNs had synergistic interaction effects with 10 Gy X-ray irradiation on increasing tumor cells AI and necrosis, and decreasing MVD. These results provided evidence that HIF-1 $\alpha$ and GLUT-1 may be promising targets for increasing tumor radiosensitivity, however, the interplay among radioresistance, HIF-1 and GLUT-1 expression warrants further investigation.GLUT1 and HIF- $1 \alpha$ mRNA, and protein levels showed significant linear correlations, however, neither GLUT1 nor HIF-1 $\alpha$ expression was correlated significantly with SUVmaxT/N. ${ }^{18} \mathrm{~F}-\mathrm{FDG}$ SUVmaxT/N apparently had no value in assessing the therapeutic results of xenografts, because of the large variation in FDG tumor uptake. In the future, we will use more mice in our experiments and apply gene silencing methods potentially useful for developing new personalized therapeutic strategies for patients with laryngeal carcinoma. We will also use PET/CT with other radiotracer for further evaluation of the therapeutic effects.

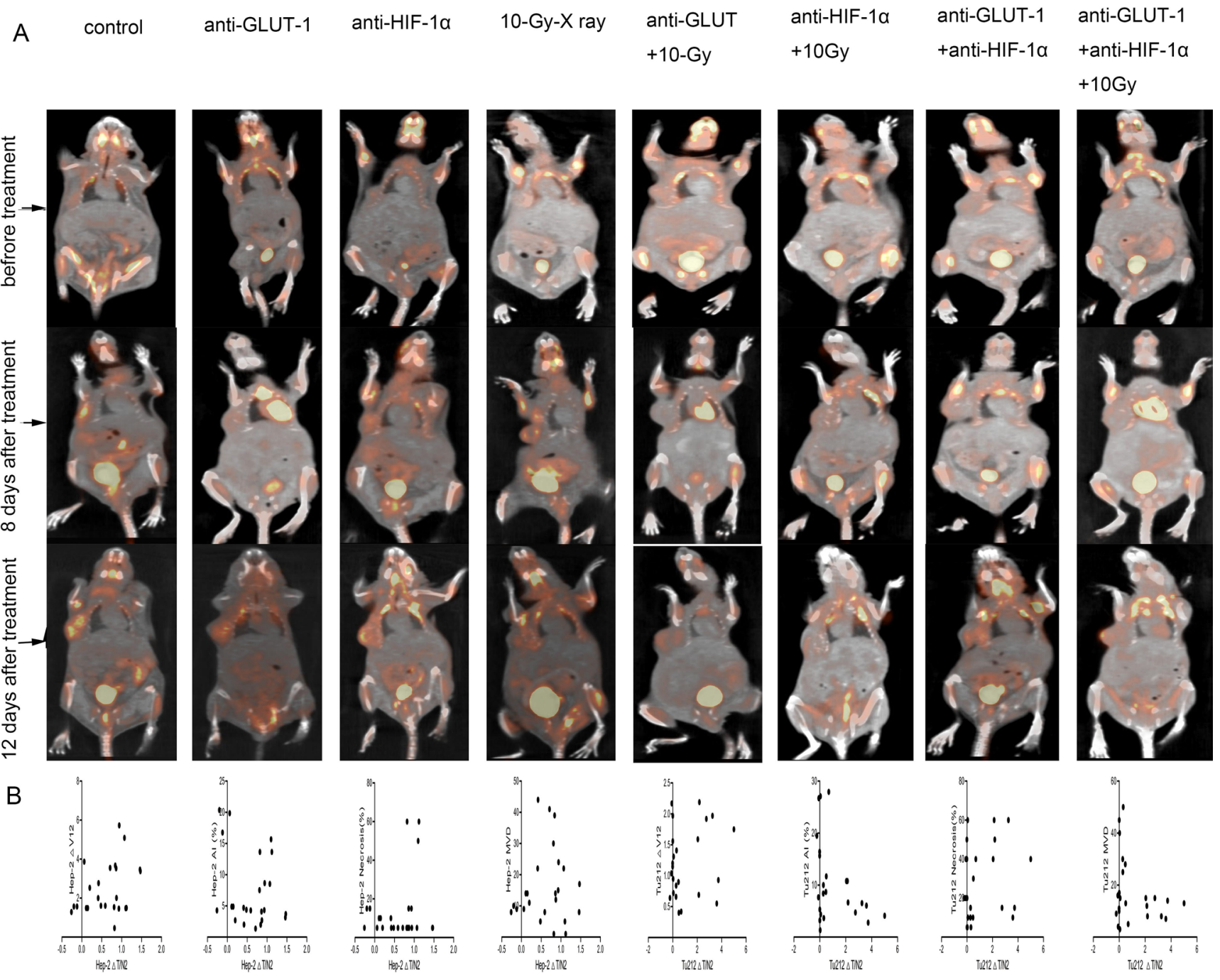

Figure 9: Tumors developed in the right flanks of mice (A). In both the Hep-2 and Tu212 groups (B), $\Delta$ SUVmaxT/N2 showed no statistical correlation with $\Delta \mathrm{V} 12$, necrosis, apoptosis or MVD (all $p>0.05$ ). 


\section{MATERIALS AND METHODS}

\section{Ethics statement}

Investigation has been conducted in accordance with the ethical standards and according to the Declaration of Helsinki and according to national and international guidelines and has been approved by the authors' institutional review board.

\section{Reagents}

The Hep-2 and Tu212 cell lines were purchased from the Cell Research Institute of Chinese Academy of Sciences (Shanghai, China) and XiangYa Central Experiment Laboratory (Hunan, China), respectively. Trypsin-EDTA solution and Lipofectamine 2000 were from Invitrogen Co. Ltd. USA. Sequences of the entire coding regions of GLUT-1 and HIF- $1 \alpha$ were obtained from GenBank, and primers were designed using ClustalX and the Omega 2.0 software. CD34 was purchased from Proteintech (Chicago, IL, USA, Catalog No: 14486-1-AP). Four-week-old female nude mice (BALB/c AnNCrj-nu/nu) weighted 15-19 g were from Shanghai Sippr-BK Laboratory Animal Co. Ltd.

\section{Cell culture}

Hep-2 and Tu212 cells were both cultured in RPMI-1640 (Gibco-BRL, Gaithersburg, MD, USA) supplemented with $10 \%$ heat-inactivated fetal bovine serum (Hyclone, Logan, UT, USA), $100 \mathrm{U} / \mathrm{mL}$ penicillin and $100 \mathrm{~g} / \mathrm{mL}$ streptomycin at $37^{\circ} \mathrm{C}$ in a $5 \% \mathrm{CO}_{2}$ atmosphere. Cells were trypsinised and harvested after reaching $80-90 \%$ confluence.

\section{Preparation of GLUT-1 AS-ODNs and HIF-10 AS-ODNs}

After pcDNA3.1 plasmids (Xunjie biological technology Co. Ltd, Hangzhou, China) were digested with HindIII and XbaI, anti-GLUT-1 cDNA or anti-HIF-1 $\alpha$ cDNA was subcloned into the vector pcDNA3.1 using T4 DNA ligase. The sequence of the GLUT-1 AS-ODN is 5'-ACAGAAAAGATGGCCACTGAG-3' and HIF- $1 \alpha$ AS-ODN is 5'-GCCGGCGCCCTCCAT-3'. The products were transfected into xenograft tumors using Lipofectamine 2000 reagent.

\section{Experimental design}

The optimization of formulation variables was carried out using $2^{3}$ factorial design. Three independent formulation variables such as GLUT-1 AS-ONDs, HIF-1 $\alpha$ AS-ODNs and X-ray irradiation each at two coded levels were designed., GLUT-1 AS-ODNs and HIF-1 $\alpha$ AS-ODNs $100 \mu \mathrm{g}$ or $0 \mu \mathrm{g}$, X-ray irradiation $10 \mathrm{~Gy}$ or
0 Gy. The effect of these factors was studied on tumor volume, tumor weight, MVD, AI, necrosis of the formulation as response variables.

\section{Nude mouse xenograft model}

This experiment was performed in accordance with the institutional guidelines of the First Affiliated Hospital, College of Medicine, Zhejiang University. The animals were housed in a specific pathogen-free room under controlled temperature and humidity. Approximately $2 \times 10^{7} / \mathrm{mL} \times 0.2 \mathrm{~mL}$ of Hep- 2 cells or Tu212 cells were inoculated subcutaneously into the right flank of mice. After 1 week, a grain-sized induration developed at the inoculation site, confirming that the xenograft model was established successfully. The mental state, food intake and activity of the mice were monitored daily. Micro PET/ $\mathrm{CT}$ and X-ray radiation were performed under general anesthesia ( $7 \mathrm{ml} / \mathrm{kg}$ ip injection of $4 \%$ chloral hydrate).

The independent variables investigated were GLUT-1 AS-ODNs, HIF-1 $\alpha$ AS-ODNs, and 10 Gy X-ray irradiation. The Hep-2 $(n=48)$ and Tu212 $(n=48)$ groups were treated as follows: (a) GLUT-1 AS-ODNs, HIF-1 $\alpha$ AS-ODNs, and 10Gy X-ray irradiation combination group, the mice were injected peritumorally with $100 \mu \mathrm{g}$ GLUT-1 AS-ODNs three times at 3-day intervals (on day 1 , day 4 , day 7), the tumors were exposed to 10 Gy X-ray once on day 5, and then $100 \mu \mathrm{g}$ HIF-1 $\alpha$ AS-ODNs was injected peritumorally into the tumors three times at $24 \mathrm{~h}$ intervals (on day5 immediately after irradiation, on day 6, day 7); (b) irradiation group, tumors were exposed to 10 Gy X-ray once on day 5; (c) GLUT-1 AS-ODNs group, the tumors were injected with GLUT1 AS-ODNs on day 1 , day 4, day 7 as above; (d) HIF-1 $\alpha$ AS-ODNs group, the tumors were injected with HIF-1 $\alpha$ AS-ODNs on day 5, day 6, day 7 as above; (e) GLUT-1 AS-ODNs and irradiation combination group, the tumors were injected with GLUT-1 AS-ODNs and irradiated as above; (f) HIF-1 $\alpha$ AS-ODNs and irradiation combination group, the tumors were injected with HIF-1 $\alpha$ AS-ODNs and irradiated as above; (g) GLUT-1 AS-ODNs and HIF$1 \alpha$ AS-ODNs combination group, the tumors were injected with GLUT-1 AS-ODNs and HIF-1 $\alpha$ AS-ODNs as above; (h) control group, the tumors were injected with RPMI1640 on corresponding days. In each group, we randomly selected three mice to undergo microPET/CT three times (on day 0 before treatment, on day 8 and day 12 after treatment initiation). Mice that didn't undergo microPET/ CT were sacrificed on day 8, others were sacrificed on day 12 , these tumors were harvested and stored at $-80^{\circ} \mathrm{C}$.

\section{Xenograft volume}

Tumor volumes were calculated every two days, based on caliper measurements of the short (a) and long (b) tumor diameters. Tumor volumes were calculated 
according to the formula $\mathrm{V}=1 / 2 \times \mathrm{a}^{2} \times \mathrm{b}$. The change in xenograft volume on day $\mathrm{x}$ was defined as $\Delta \mathrm{Vx}$, $\Delta \mathrm{Vx}=(\mathrm{Vx}-\mathrm{V} 0) / \mathrm{V} 0$, where $\mathrm{Vx}$ is xenograft volume on day $\mathrm{x}$, and $\mathrm{V} 0$ is the xenograft volume on day 0 .

\section{Micro PET/CT}

${ }^{18} \mathrm{~F}-\mathrm{FDG}$ was synthesized by the PET Centre at our hospital. The animals were fasted overnight. ${ }^{18} \mathrm{~F}-\mathrm{FDG}$ $(250 \mu \mathrm{Ci}$ in $0.2 \mathrm{~mL})$ was injected via the tail vein prior to PET scanning. The animals were anesthetized before PET/ CT scanning. Then $1 \mathrm{~h}$ later PET scanning was performed. Mice were placed prone in the center of Siemens Inveon combined microPET-CT scanner (Siemens Preclinical Solution USA, Inc., Knoxville, TN, USA) with limbs stretched. MicroCT scans were performed with an X-ray tube voltage of $80 \mathrm{kV}$, a current of $500 \mu \mathrm{A}$, an exposure time of $150 \mathrm{~ms}$, and 120 rotation steps. A $10 \mathrm{~min}$ PET static acquisition was performed and the images were reconstructed using OSEM (ordered set expectation maximization) algorithm for 3D PET reconstruction. Images were analyzed with the Inveon Research Workplace 4.1 (Siemens, Erlangen, Germany). The standardized uptake value (SUV, the unit of SUV is $\mathrm{g} / \mathrm{ml}$ ) was determined by the formula $\mathrm{SUV}=[(\mathrm{RTA} / \mathrm{cm} 3) /$ RID] $\times \mathrm{BW}$, where RTA is the measured radiotracer tissue activity (mCi), RID is the radiotracer injected dose $(\mathrm{mCi})$, and BW is the mouse body weight $(\mathrm{g})$. The maximum SUV (SUVmax) of the tumor (SUVmaxT) and opposite normal subcutaneous tissue (SUVmaxN) were recorded. SUVmaxT/ $\mathrm{N}=\mathrm{SUVmaxT} / \mathrm{SUVmaxN}, \mathrm{SUVmax} \mathrm{T} / \mathrm{N}$ on day 0 , day 8 and day 12 were referred as SUVmax T/N0, SUVmax T/N1, SUVmax T/N2, respectively. The change in ${ }^{18} \mathrm{~F}$-FDG uptake for each tumor was determined using the following equations: $\Delta \mathrm{SUVmaxT} / \mathrm{N} 1=(\mathrm{SUV} \operatorname{maxT} / \mathrm{N} 1-\mathrm{SUV} \operatorname{maxT} /$ N0)/SUVmaxT/N0, $\Delta \mathrm{SUVmaxT} / \mathrm{N} 2=(\mathrm{SUV} \operatorname{maxT} / \mathrm{N} 2-$ SUVmaxT/N0)/SUVmaxT/N0, $\Delta \mathrm{SUV}_{\max } \mathrm{T} / \mathrm{N} 12=$ (SUVmax T/N2- SUVmax T/N1)/ SUVmax T/N1.

\section{Reverse transcription polymerase chain $\operatorname{reaction}(\mathbf{R T}-\mathrm{PCR})$}

Tumor tissue homogenates were collected and added Trizol according to the manufacturer's protocol, RNA was isolated and reverse transcribed. First,4.2 $\mu \mathrm{g}$ RNA, $2 \mu \mathrm{L}$ Oligo(dT) $(10 \mu \mathrm{M}), 2 \mu \mathrm{L}$ dNTP $(2.5 \mathrm{mM})$ were mixed and $\mathrm{ddH}_{2} \mathrm{O}$ (RNase free) was added up to $14.5 \mu \mathrm{L}$. Reactions were incubated at $70^{\circ} \mathrm{C}$ for 5 min and kept on ice after centrifuging briefly, and then $5 \times$ RT buffer $(4 \mu \mathrm{l})$, HRP(RRI)/RNase inhibitor $(0.5 \mu \mathrm{l})$, and M-MLV $(1 \mu \mathrm{L})$ were added. After gentle mixing, the tubes were incubated at $42^{\circ} \mathrm{C}$ for $60 \mathrm{~min}, 95^{\circ} \mathrm{C}$ for $5 \mathrm{~min}$. The PCR reagents included $10 \mu \mathrm{L} 2 \times$ real-time PCR master mix (SYBR ${ }^{\circledR}$-Green), $2 \mu \mathrm{L}$ dNTP $(2.5 \mathrm{mM}), 0.25 \mu \mathrm{L}$ Ex Taq, $2.5 \mu \mathrm{L} 10 \times$ Ex Taq E buffer, $1 \mu \mathrm{L}$ cDNA, $9.25 \mu \mathrm{L}$ ddH2O, in a total volume of $25 \mu \mathrm{L}$. The PCR primers used were as follows:
GAPDH sense, 5'-TGTTGCCATCAATGACCCCTT-3', GAPDH antisense, 5'-CTCCACGACGTACTCAGCG-3' (202 bp), GLUT-1 sense, 5'-GTCAACACGGCCTTCACTG-3', GLUT-1 antisense, 5'-GGTCATGAGTATGGCACAACC-3' (111 bp), HIF-1 $\alpha$ sense, 5 '-TTACAGCAGCCAGACGATCA-3', HIF-1 $\alpha$ antisense, 5'-CCCTGCAGTAGGTTTCTGCT-3' (233 bp). To calculation differential gene expression, the 2$\Delta \Delta \mathrm{Ct}$ formula was used.

\section{Western blotting}

Tumor tissue homogenates were lysed in Radio Immunoprecipitation Assay (RIPA) lysis solution and were separated by gel electrophoresis and transferred to membranes. The membranes were blocked with $5 \%$ non-fat dry milk in TBST and then soaked in the primary antibody buffer, overnight at $4^{\circ} \mathrm{C}$ (GLUT-1 1:800 dilution(Proteintech, Chicago, IL, USA, cat no: 21829-1AP), (HIF-1 $\alpha$ 1:800 dilution (Proteintech, Chicago, IL, USA, cat no: 20960-1-AP). The membranes were soaked in secondary antibody buffer and incubated for $2 \mathrm{~h}$ at room temperature. The proteins were visualized using enhanced chemiluminescence and exposed to X-ray film. Protein expression was analyzed semi-quantitatively using the Gel Logic analysis system (Kodak, Rochester, NY, USA).

\section{Analysis of MVD}

Sections were cut at $5 \mu \mathrm{m}$ and placed on a Fisher Superfrost slide and dried for $1-2 \mathrm{~h}$ at room temperature. Slides were fixed with $4 \%$ polyoxymethylene and incubated with PBS containing $0.5 \%$ Triton X-100 successively to improve the penetration of the antibody. Primary antibody(CD34) was dropped on the coverslips and incubated overnight at $4^{\circ} \mathrm{C}$ according to the manufacturer's protocol. The slides were observed using a microscope, positive signals were pale brown or brown. To quantify the MVD, an area with maximum concentration of vessels was identified at low magnification $(\times 100)$ and the three most intense fields were chosen for blood vessel counts at $\times 200$ magnification. The mean of the three counts was calculated.

\section{Transferase-mediated dUTP digoxigenin nick end-labeling (TUNEL)}

Tumor sections were assessed using the In Situ Cell Death Detection Kit-POD (Roche, Shanghai, China). Tissue sections were fixed using fixation solution and incubated with blocking solution. TUNEL reaction mixture was added according to the manufacturer's protocol. Staining was visualized under an optical microscope. Cells in which the nuclei were brown or brown-yellow were considered as positive. The total number of apoptotic cells at $\times 400$ magnification in five randomly selected fields was counted. The AI was calculated as the percentage of positively stained cells, $\mathrm{AI}=$ number of apoptotic cells $\times 100 /$ total number of nucleated cells. 


\section{Haematoxylin and eosin (H\&E)}

Microscope slides with rehydrated tumor sections were prepared and dipped into a Coplin jar containing Mayer's hematoxylin for 30s, rinsed in $\mathrm{H}_{2} \mathrm{O}$ for $1 \mathrm{~min}$, and stained with $1 \%$ eosin Y solution for 10-30s. Sections were dehydrated using two changes of $95 \%$ alcohol and two changes of $100 \%$ alcohol for 30 s each. Each slice was assessed in three random microscopic fields $(\times 400)$. The necrosis rate $=$ necrosis area/total area in the field.

\section{Statistical analysis}

Data were analyzed using SPSS software (ver 22.0). A $2^{3}$ factorial design was adopted in this study concerning with the effects of formulation variables and their interactions on response variables to obtain the optimized formulation. Pearson's analysis was used to evaluate the relationships among GLUT-1 and HIF-1 $\alpha$ expression, ${ }^{18} \mathrm{~F}-\mathrm{FDG}$ accumulation and therapeutic effects. $P$ values $<0.05$ were considered to indicate statistical significance.

\section{Abbreviations}

HIF-1 $\alpha$ : hypoxia-inducible factor $1 \alpha$; GLUT-1: glucose transporter-1; ${ }^{18} \mathrm{~F}-\mathrm{FDG}$ : 2'-deoxy-2'-[18F] fluoro-D-glucose; PET/CT: positron emission tomography-computed tomography; AS-ODNs: antisense oligodeoxynucleotides; MVD: microvessel density; AI: apoptosis index; SUV: standardized uptake value; RT-PCR: reverse transcription polymerase chain reaction.

\section{Authors' contributions}

Li-Fang Shen and Shui-Hong Zhou designed the experiments; Li-Fang Shen, Xin Zhao, Zhong-Jie Lu, KuiZhao, Jun Fan, Min-Li Zhou carried out the experiments and participated in result interpretation; Li-Fang Shen and Shui-Hong Zhou wrote the manuscript. All authors revised the manuscript.

\section{ACKNOWLEDGMENTS}

We acknowledge the assistance of all those who participated in this project, especially the technologists in the PET department of the First Affiliated Hospital, College of Medicine, Zhejiang University.

\section{CONFLICTS OF INTEREST}

The authors declare that they have no conflicts of interest.

\section{FUNDING}

This research was supported by National Natural Science Foundation of China (No. 81172562 and
81372903), and Science and Technology Department of Zhejiang Province, China (No.2016C33144).

\section{Ethical approval}

All procedures performed in studies involving mice were in accordance with in accordance with institutional guidelines of the First Affiliated Hospital, College of Medicine, Zhejiang University and with appropriate institutional certification.

\section{Informed consent}

Informed consent was obtained from all individual participants included in the study.

\section{REFERENCES}

1. Siegel R, Ma J, Zou Z, Jemal A. Cancer statistics, 2014. CA Cancer J Clin. 2014; 64:9-29.

2. Fang J, Zhou SH, Fan J, Yan SX. Roles of glucose transporter-1 and the phosphatidylinositol 3-kinase/protein kinase B pathway in cancer radioresistance. Molecular Medicine Reports. 2015; 11:1573-81.

3. Perri F, Pacelli R, Scarpati GD, Cella L, Giuliano M, Caponigro F, Pepe S. Radioresistance in head and neck squamous cell carcinoma: Biological bases and therapeutic implications. Head And Neck. 2015; 37:763-70.

4. Wang MX, Li XM, Qu YT, Xu O, Sun QJ. Hypoxia promotes radioresistance of CD133-positive Hep-2 human laryngeal squamous carcinoma cells in vitro. International Journal Of Oncology. 2013; 43:131-40.

5. Roh JL, Cho KJ, Kwon GY, Ryu CH, Chang HW, Choi SH, Nam SY, Kim SY. The prognostic value of hypoxia markers in T2-staged oral tongue cancer. Oral Oncol. 2009; 45:63-8.

6. Isa AY, Ward TH, West CM, Slevin NJ, Homer JJ. Hypoxia in head and neck cancer. Br J Radiol. 2006; 79:791-8.

7. Rankin EB, Giaccia AJ. The role of hypoxia-inducible factors in tumorigenesis. Cell Death Differ. 2008; 15:678-85.

8. Wu XH, Chen SP, Mao JY, Ji XX, Yao HT, Zhou SH. Expression and significance of hypoxia-inducible factor1alpha and glucose transporter-1 in laryngeal carcinoma. Oncol Lett. 2013; 5:261-6.

9. Xiang ZL, Zeng ZC, Fan J, Tang ZY, He J, Zeng HY, Chang JY. The expression of HIF-1alpha in primary hepatocellular carcinoma and its correlation with radiotherapy response and clinical outcome. Mol Biol Rep. 2012; 39: 2021-9.

10. Jin Z, Aixi Y, Baiwen Q, Zonghuan L, Xiang H. Inhibition of hypoxia-inducible factor-1 alpha radiosensitized MG-63 human osteosarcoma cells in vitro. Tumori. 2015; 101:578-84.

11. Luo Z, Bai M, Xiao X, Zhang W, Liu X, Yang X, Li S, Huan Y, Wu Z, Zhang X, Cao W. Silencing of HIF-1alpha 
enhances the radiation sensitivity of human glioma growth in vitro and in vivo. Neuropharmacology. 2015; 89:168-74.

12. Moon SY, Chang HW, Roh JL, Kim GC, Choi SH, Lee SW, Cho KJ, Nam SY, Kim SY. Using YC-1 to overcome the radioresistance of hypoxic cancer cells. Oral Oncology. 2009; 45:915-9.

13. Lee HJ, Yoon C, Park DJ, Kim YJ, Schmidt B, Lee YJ, Tap WD, Eisinger-Mathason TS, Choy E, Kirsch DG, Simon MC, Yoon SS. Inhibition of vascular endothelial growth factor A and hypoxia-inducible factor 1alpha maximizes the effects of radiation in sarcoma mouse models through destruction of tumor vasculature. Int J Radiat Oncol Biol Phys. 2015; 91:621-30.

14. Luo XM, Zhou SH, Fan J. Glucose transporter-1 as a new therapeutic target in laryngeal carcinoma. J Int Med Res. 2010; 38:1885-92.

15. Rademakers SE, Lok J, van der Kogel AJ, Bussink J, Kaanders JH. Metabolic markers in relation to hypoxia; staining patterns and colocalization of pimonidazole, HIF1alpha, CAIX, LDH-5, GLUT-1, MCT1 and MCT4. BMC Cancer. 2011; 11:167.

16. Kunkel M, Moergel M, Stockinger M, Jeong JH, Fritz G, Lehr HA, Whiteside TL. Overexpression of GLUT-1 is associated with resistance to radiotherapy and adverse prognosis in squamous cell carcinoma of the oral cavity. Oral Oncol. 2007; 43:796-803.

17. Korkeila E, Jaakkola PM, Syrjanen K, Pyrhonen S, Sundstrom J. Pronounced tumour regression after radiotherapy is associated with negative/weak glucose transporter-1 expression in rectal cancer. Anticancer Res. 2011; 31:311-5.

18. Zhou SH, Fan J, Chen XM, Cheng KJ, Wang SQ. Inhibition of cell proliferation and glucose uptake in human laryngeal carcinoma cells by antisense oligonucleotides against glucose transporter-1. Head Neck. 2009; 31:1624-33.

19. Yan SX, Luo XM, Zhou SH, Bao YY, Fan J, Lu ZJ, Liao XB, Huang YP, Wu TT, Wang QY. Effect of antisense oligodeoxynucleotides glucose transporter-1 on enhancement of radiosensitivity of laryngeal carcinoma. Int J Med Sci. 2013; 10:1375-86.

20. Amann T, Hellerbrand C. GLUT1 as a therapeutic target in hepatocellular carcinoma. Expert Opin Ther Targets. 2009; 13:1411-27.

21. Molthoff CF, Klabbers BM, Berkhof J, Felten JT, van Gelder $\mathrm{M}$, Windhorst $\mathrm{AD}$, Slotman BJ, Lammertsma AA. Monitoring response to radiotherapy in human squamous cell cancer bearing nude mice: comparison of $2^{\prime}$-deoxy-2'-[18F]fluoro-D-glucose (FDG) and 3'-[18F]fluoro-3'-deoxythymidine (FLT). Mol Imaging Biol. 2007; 9:340-7.

22. Phelps ME. Positron emission tomography provides molecular imaging of biological processes. Proc Natl Acad Sci USA. 2000; 97:9226-33.

23. Zhao K, Yang SY, Zhou SH, Dong MJ, Bao YY, Yao HT. Fluorodeoxyglucose uptake in laryngeal carcinoma is associated with the expression of glucose transporter-1 and hypoxia-inducible-factor-1alpha and the phosphoinositide 3-kinase/protein kinase B pathway. Oncol Lett. 2014; 7:984-90.

24. Mason NS, Lopresti BJ, Ruszkiewicz J, Dong X, Joyce S, Leef G, Sen M, Wahed AS, Mathis CA, Grandis JR, Thomas SM. Utility of 3'-[(18)F]fluoro-3'-deoxythymidine as a PET tracer to monitor response to gene therapy in a xenograft model of head and neck carcinoma. Am J Nucl Med Mol Imaging. 2013; 3:16-31.

25. Zheng Y, Yang Z, Zhang Y, Shi Q, Bao X, Zhang J, Yuan H, Yao Z, Hu C, Zhang Y. The preliminary study of 18F-FLT micro-PET/CT in predicting radiosensitivity of human nasopharyngeal carcinoma xenografts. Ann Nucl Med. 2015; 29:29-36.

26. Park SS, Chunta JL, Robertson JM, Martinez AA, Oliver Wong CY, Amin M, Wilson GD, Marples B. MicroPET/ CT imaging of an orthotopic model of human glioblastoma multiforme and evaluation of pulsed low-dose irradiation. Int J Radiat Oncol Biol Phys. 2011; 80:885-92.

27. Yang X, Yang B, Cai J, Zhang C, Zhang Q, Xu L, Qin Q, Zhu H, Ma J, Tao G, Cheng H, Sun X. Berberine enhances radiosensitivity of esophageal squamous cancer by targeting HIF-1alpha in vitro and in vivo. Cancer Biol Ther. 2013; 14:1068-73.

28. Zhang C, Yang X, Zhang Q, Yang B, Xu L, Qin Q, Zhu H, Liu J, Cai J, Tao G, Ma J, Ge X, Zhang S, et al. Berberine radiosensitizes human nasopharyngeal carcinoma by suppressing hypoxia-inducible factor-1alpha expression. Acta Otolaryngol. 2014; 134:185-92.

29. Tennant DA, Duran RV, Gottlieb E. Targeting metabolic transformation for cancer therapy. Nat Rev Cancer. 2010; 10:267-77.

30. Chen T, Ren Z, Ye LC, Zhou PH, Xu JM, Shi Q, Yao LQ, Zhong YS. Factor inhibiting HIF1alpha (FIH-1) functions as a tumor suppressor in human colorectal cancer by repressing HIF1alpha pathway. Cancer Biol Ther. 2015; 16:244-52.

31. Fan G, Bo J, Wan R, Peng M, Luan Y, Deng M, Xu L. The effect of lentiviral vector-mediated RNA interference targeting hypoxia-inducible factor 1alpha on the uptake of fluorodeoxyglucose ((18)f) in the human pancreatic cancer cell line, patu8988. Cancer Biother Radiopharm. 2015; 30:160-8.

32. Meijer TW, Kaanders JH, Span PN, Bussink J. Targeting hypoxia, HIF-1, and tumor glucose metabolism to improve radiotherapy efficacy. Clin Cancer Res. 2012; 18:5585-94.

33. Song B, Shu Y, Cui T, Fu P. Allicin inhibits human renal clear cell carcinoma progression via suppressing HIF pathway. Int J Clin Exp Med. 2015; 8:20573-80.

34. Li X, Liu X, Xu Y, Liu J, Xie M, Ni W, Chen S. KLF5 promotes hypoxia-induced survival and inhibits apoptosis in non-small cell lung cancer cells via HIF-1alpha. Int J Oncol. 2014; 45:1507-14.

35. Sato M, Hirose K, Kashiwakura I, Aoki M, Kawaguchi H, Hatayama Y, Akimoto H, Narita Y, Takai Y. LW6, a 
hypoxia-inducible factor 1 inhibitor, selectively induces apoptosis in hypoxic cells through depolarization of mitochondria in A549 human lung cancer cells. Mol Med Rep. 2015; 12:3462-8.

36. Shin SJ, Kim JY, Kwon SY, Mun KC, Cho CH, Ha E. Ciglitazone enhances ovarian cancer cell death via inhibition of glucose transporter-1. Eur J Pharmacol. 2014; 743:17-23.

37. Shiau AL, Shen YT, Hsieh JL, Wu CL, Lee CH. Scutellaria barbata inhibits angiogenesis through downregulation of HIF-1 alpha in lung tumor. Environ Toxicol. 2014; 29:363-70.

38. Zeng L, Morinibu A, Kobayashi M, Zhu Y, Wang X, Goto Y, Yeom CJ, Zhao T, Hirota K, Shinomiya K, Itasaka S, Yoshimura M, Guo G, et al. Aberrant IDH3alpha expression promotes malignant tumor growth by inducing HIF-1mediated metabolic reprogramming and angiogenesis. Oncogene. 2015; 34:4758-66.

39. Iwasaki K, Yabushita H, Ueno T, Wakatsuki A. Role of hypoxia-inducible factor-1alpha, carbonic anhydrase-IX, glucose transporter-1 and vascular endothelial growth factor associated with lymph node metastasis and recurrence in patients with locally advanced cervical cancer. Oncol Lett. 2015; 10:1970-8.

40. Yin T, He S, Shen G, Wang Y. HIF-1 Dimerization Inhibitor Acriflavine Enhances Antitumor Activity of Sunitinib in Breast Cancer Model. Oncol Res. 2014; 22:139-45.

41. Liu Y, Cao Y, Zhang W, Bergmeier S, Qian Y, Akbar H, Colvin R, Ding J, Tong L, Wu S, Hines J, Chen X. A smallmolecule inhibitor of glucose transporter 1 downregulates glycolysis, induces cell-cycle arrest, and inhibits cancer cell growth in vitro and in vivo. Mol Cancer Ther. 2012; 11:1672-82.

42. Petty JC, Lana SE, Thamm DH, Charles JB, Bachand AM, Bush JM, Ehrhart EJ. Glucose transporter 1 expression in canine osteosarcoma. Vet Comp Oncol. 2008; 6:133-40.

43. Miyawaki A, Ikeda R, Hijioka H, Ishida T, Ushiyama M, Nozoe E, Nakamura N. SUVmax of FDG-PET correlates with the effects of neoadjuvant chemoradiotherapy for oral squamous cell carcinoma. Oncol Rep. 2010; 23:1205-12.

44. Takebayashi R, Izuishi K, Yamamoto Y, Kameyama R, Mori H, Masaki T, Suzuki Y. [18F]Fluorodeoxyglucose accumulation as a biological marker of hypoxic status but not glucose transport ability in gastric cancer. J Exp Clin Cancer Res. 2013; 32:34.

45. Jo MS, Choi OH, Suh DS, Yun MS, Kim SJ, Kim GH, Jeon HN. Correlation between expression of biological markers and [F]fluorodeoxyglucose uptake in endometrial cancer. Oncol Res Treat. 2014; 37:30-4.

46. Mees G, Dierckx R, Vangestel C, Laukens D, Van Damme N, Van de Wiele C. Pharmacologic activation of tumor hypoxia: a means to increase tumor 2-deoxy-2-[18F] fluoro-D-glucose uptake? Mol Imaging. 2013; 12:49-58.

47. Yamada T, Uchida M, Kwang-Lee K, Kitamura N, Yoshimura T, Sasabe E, Yamamoto T. Correlation of metabolism/hypoxia markers and fluorodeoxyglucose uptake in oral squamous cell carcinomas. Oral Surg Oral Med Oral Pathol Oral Radiol. 2012; 113:464-71.

48. Krak NC, Boellaard R, Hoekstra OS, Twisk JW, Hoekstra CJ, Lammertsma AA. Effects of ROI definition and reconstruction method on quantitative outcome and applicability in a response monitoring trial. Eur J Nucl Med Mol Imaging. 2005; 32:294-301.

49. Boellaard R, Krak NC, Hoekstra OS, Lammertsma AA. Effects of noise, image resolution, and ROI definition on the accuracy of standard uptake values: a simulation study. J Nucl Med. 2004; 45:1519-27.

50. Weber WA. Assessing tumor response to therapy. J Nucl Med. 2009; 50:1S-10S.

51. Uzel EK, Ekmekcioglu O, Elicin O, Halac M, Uzel OE. Is FDG -PET-CT a valuable tool in prediction of persistent disease in head and neck cancer. Asian Pac J Cancer Prev. 2013; 14:4847-51.

52. Plathow C, Weber WA. Tumor cell metabolism imaging. J Nucl Med. 2008; 49:43S-63S.

53. Zhao S, Kuge Y, Mochizuki T, Takahashi T, Nakada K, Sato M, Takei T, Tamaki N. Biologic correlates of intratumoral heterogeneity in $18 \mathrm{~F}-\mathrm{FDG}$ distribution with regional expression of glucose transporters and hexokinaseII in experimental tumor. J Nucl Med. 2005; 46:675-82.

54. Pugachev A, Ruan S, Carlin S, Larson SM, Campa J, Ling CC, Humm JL. Dependence of FDG uptake on tumor microenvironment. Int J Radiat Oncol Biol Phys. 2005; 62:545-53.

55. Wang H, Liu B, Tian J, Xu B, Zhang J, Qu B, Chen Y. Evaluation of (1)(8)F-FDG and (1)(8)F-FLT for monitoring therapeutic responses of colorectal cancer cells to radiotherapy. Eur J Radiol. 2013; 82:e484-91.

56. Hong JH, Kim HH, Han EJ, Byun JH, Jang HS, Choi EK, Kang JH, Yoo Ie R. Total Lesion Glycolysis Using (1)(8) F-FDG PET/CT as a Prognostic Factor for Locally Advanced Esophageal Cancer. J Korean Med Sci. 2016; 31:39-46. 